\title{
Non-Abelian anomalies in multi-Weyl semimetals
}

\author{
Renato M. A. Dantas $\odot{ }^{1, *}$ Francisco Peña-Benitez, ${ }^{1, \dagger}$ Bitan Roy, ${ }^{1,2, \ddagger}$ and Piotr Surówka ${ }^{1, \S}$ \\ ${ }^{1}$ Max-Planck-Institut für Physik komplexer Systeme, Nöthnitzer Straße 38, 01187 Dresden, Germany \\ ${ }^{2}$ Department of Physics, Lehigh University, Bethlehem, Pennsylvania 18015, USA
}

(Received 2 August 2019; published 3 January 2020)

\begin{abstract}
We construct the effective field theory for time-reversal symmetry-breaking multi-Weyl semimetals (MWSMs), composed of a single pair of Weyl nodes of (anti)monopole charge $n$, with $n=1,2,3$ in crystalline environment. From both the continuum and lattice models, we show that a MWM with $n>1$ can be constructed by placing $n$ flavors of linearly dispersing simple Weyl fermions (with $n=1$ ) in a bath of an SU(2) non-Abelian static background gauge field. Such an SU(2) field preserves certain crystalline symmetry (fourfold rotational or $C_{4}$ in our construction), but breaks the Lorentz symmetry, resulting in nonlinear band spectra, namely, $E \sim\left(p_{x}^{2}+p_{y}^{2}\right)^{n / 2}$, but $E \sim\left|p_{z}\right|$, for example, where momenta $\mathbf{p}$ is measured from the Weyl nodes. Consequently, the effective field theory displays $\mathrm{U}(1) \times \mathrm{SU}(2)$ non-Abelian anomalies, yielding the anomalous Hall effect, its non-Abelian generalization, and various chiral conductivities. The anomalous violation of conservation laws is determined by the monopole charge $n$ and a specific algebraic property of the SU(2) Lie group, which we further substantiate by numerically computing the regular and isospin densities from the lattice models of MWSMs. These predictions are also supported from a strongly coupled (holographic) description of MWSMs. Altogether our findings unify the field-theoretic descriptions of MWSMs of arbitrary monopole charge $n$ (featuring $n$ copies of the Fermi arc surface states), predict signatures of non-Abelian anomaly in table-top experiments, and pave the way to explore the structure of anomalies for multifold fermions, transforming under arbitrary half-integer or integer spin representations.
\end{abstract}

DOI: 10.1103/PhysRevResearch.2.013007

\section{INTRODUCTION}

Anomalies are traditionally studied in the realm of relativistic field theories that are pertinent in high-energy physics [1-8]. They show up as the violation of symmetries of the classical action upon quantization of chiral massless fermions. An intrinsic feature of high-energy theories is that they are Lorentz symmetric, stemming from the linear dispersion of the chiral fermions. Also in the world of condensed-matter systems an emergent relativistic symmetry results from the quasiparticle spectra that are linear in momentum, but at low energies. This is the quintessential feature of Weyl semimetals, a class of materials where quantum anomaly has been studied theoretically [9-16] and its signature has possibly been observed in experiments [17-27].

More intriguingly, condensed-matter systems offer unique opportunities to further extend our understanding of anomalies in quantum field theories and its connections with transport. Recent developments have allowed us to go beyond the

\footnotetext{
*rmad@pks.mpg.de

†pena@pks.mpg.de

¥bir218@lehigh.edu

§surowka@pks.mpg.de
}

Published by the American Physical Society under the terms of the Creative Commons Attribution 4.0 International license. Further distribution of this work must maintain attribution to the author(s) and the published article's title, journal citation, and DOI. original paradigm of linearly dispersing chiral fermions, as nowadays gapless chiral systems with finite band curvatures can be found in various solid-state compounds. The main motivation of our work is to pedagogically develop a comprehensive understanding of such systems, lacking the Lorentz symmetry from their effective low-energy field theory and anchor various field-theoretic predictions from concrete but simple lattice models (on a cubic lattice). One representative class of systems where such a theory should be applicable is so-called the multi-Weyl semimetals. These systems possess linear dispersion only along one component of the momentum, while displaying finite band curvature along the remaining two crystalline directions (see Fig. 1). The power-law dependence of the band dispersion $(n)$ is set by the charge $n$ of the corresponding pairs of (anti)monopole in the momentum space that act as source and sink of Abelian Berry curvature and in turn also determines the integer topological invariant of the system. Therefore, the present discussion should allow us to pave the way to connect the notion of quantum anomalies with the topological invariant of gapless chiral systems.

Our main achievements are the following. We show that multi-Weyl semimetals (with $n>1$ ) generically exhibit nonAbelian anomalies, leading to the nonconservation of isospin density. We also show that both Abelian and non-Abelian anomaly coefficients are solely determined by the topological invariant $(n)$ of the system. Notice that quantum field theories depict three types of chiral anomalies: Abelian, non-Abelian, and gravitational. While negative longitudinal magnetoresistance, bearing the signature of Abelian anomaly, has been observed in a number of Weyl materials [17-24] and some of 
them also show an indirect signature of mixed gauge-gravity anomaly in the thermal transport $[25,26,28,29]$, the presence or signature of non-Abelian anomaly has remained illusive so far. Also, the regime where high-energy systems may support non-Abelian anomalous transport has been quite challenging to access experimentally until now [6,30]. By contrast, we show here that multi-Weyl semimetals constitute the ideal platform to capture the signature of non-Abelian anomaly in table-top experiments (from the nonconservation of isospin density), (possibly) standing as the final milestone of anomaly controlled transports. Further, as a collateral consequence, in this work we liberate the notion of Abelian (or generically any) anomaly from the burden of the Lorentz invariance.

Besides the genuine fundamental importance of our quest, it should also be relevant for real materials, as Weyl points with $n=2$ (known as double-Weyl nodes) can be found in $\mathrm{HgCr}_{2} \mathrm{Se}_{4}$ [31,32] and $\mathrm{SrSi}_{2}$ [33], whereas $A(\mathrm{MoX})_{3}$ (with $A=\mathrm{Rb}$ or $\mathrm{Tl}$ and $X=\mathrm{Te}$ ) can accommodate Weyl points with $n=3$ (known as triple-Weyl nodes) [34]. Even though at a formal level our conclusions hold for any arbitrary integer value of $n$, crystalline environment forbids realization of symmetry-protected Weyl nodes with $n>3$ [35].

We note that a direct approach to construct an effective field theory for multi-Weyl semimetals has been discussed previously in Refs. [36,37], where a Lagrangian with an anisotropic energy spectrum and the corresponding anomalous violation of chiral symmetry was computed. We emphasize that this approach is cumbersome and may even be problematic for the following reasons. First of all, we stress that departure from the Lagrangian that is linear in (space-time) four-momenta changes the structure of fermionic operators and all the anomalies need to be calculated from the scratch. Next, the Lagrangian corresponding to anisotropic dispersion obscures the underlying symmetry structure (and may even hinder additional features, about which more in a moment). Finally, previous studies on Lorentz violating theories suggest that certain ambiguities may appear in the formulation that cannot be removed within the effective theory description [11]. To circumvent these pitfalls and address the structure of the anomaly in multi-Weyl systems in an unambiguous and transparent fashion, we develop here a completely different theoretical approach, highlighted below. For the sake of concreteness, we focus on the minimal model for time-reversal symmetry-breaking Weyl semimetals, composed of only a single pair of (anti)monopole of charge $n$.

Given this motivation, we construct an effective field theory for multi-Weyl systems that is always linear in all momenta, but accompanied by a Lorentz violating perturbation, which ultimately leads to the multi-Weyl spectrum in the low-energy limit (see Fig. 1). As we show, this formulation has several advantages over the direct approach and improves the analysis in every aspect mentioned above. For instance, from a computational point of view, we do not need to perform additional computations of anomalies, as the theory is always linear. Furthermore, we find that the requisite Lorentz symmetry-breaking perturbation yielding the multiWeyl spectra at low energy couples to linearly dispersing chiral fermions as a SU(2) non-Abelian constant gauge field. As a result, the structure of the anomaly is much richer than the ones inferred from previous studies [36,37]. Namely,

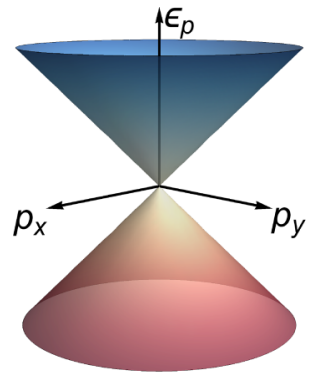

(a)

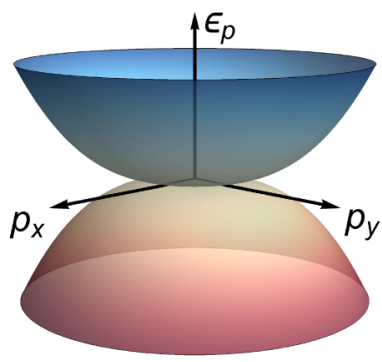

(c)

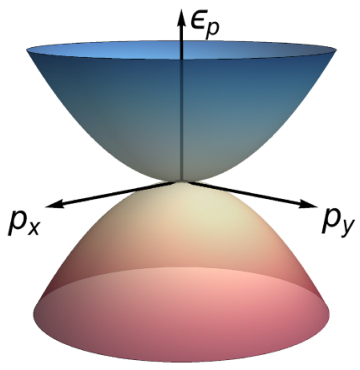

(b)

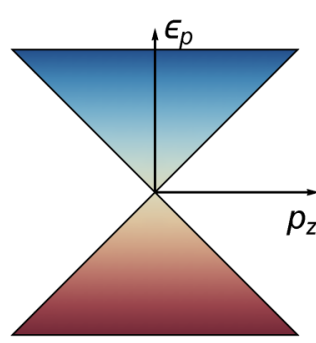

(d)
FIG. 1. Energy dispersion $\epsilon_{\mathbf{p}}$ for multi-Weyl fermions in the ( $p_{x}, p_{y}$ ) plane for (a) $n=1$, (b) $n=2$, and (c) $n=3$ around a Weyl node. For $n=1,2$, and 3 the valence and conduction bands display, respectively, linear, quadratic, and cubic touching in this plane. However, the dispersion always scales linearly with $p_{z}$ irrespective of $n$, as shown in (d). The momentum $\mathbf{p}$ is measured from the Weyl nodes, placed at $\mathbf{p}=0$.

in addition to the usual (but generalized) $\mathrm{U}(1)$ anomalies, we also unveil non-Abelian SU(2) anomalies for multi-Weyl semimetals when $n>1$. Due to the extensive nature of our study, it is worth pausing at this point to offer an overview of the main results, before delving into the details.

\section{A. Extended summary}

The minimal effective low-energy model for a multi-Weyl semimetal can be described in terms of two-component chiral (left or right) fermions (see Sec. II). The resulting quasiparticle spectra in the vicinity of each Weyl node scale as $E \sim\left|p_{z}\right|$ and $E \sim p_{\perp}^{n}$, where $p_{\perp}=\left[p_{x}^{2}+p_{y}^{2}\right]^{1 / 2}$ (see Fig. 1), since in our construction the Weyl nodes are separated along the $z$ direction. Here $n$ is an integer that determines the (anti)monopole charge of the Weyl nodes and hence the topological invariant of the system. Therefore, when $n>1$ the energy dispersion in the $x y$ plane displays nontrivial band curvature. In Sec. II, we also show that such a nonlinear dispersion for a multi-Weyl semimetal can be achieved at low energies by coupling $n$ copies of simple Weyl fermions [with $n=1$, possessing only linear dispersion (see Fig. 1)] with a $C_{4}$-symmetry-preserving perturbation $(\Delta)$. In the language of effective field theory, such a perturbation breaks the Lorentz invariance and couples with simple Weyl fermions as an SU(2) non-Abelian constant gauge field. Consequently, a multi-Weyl semimetal gets immersed in a constant nonAbelian magnetic field $\mathbf{B}_{3} \sim \Delta^{2}$. Nonetheless, these two 
constructions are shown to be equivalent as they yield identical band dispersion at low energies and the topological invariant of the system. However, the latter construction allows us to derive an effective field theory for generalized Weyl systems with $n>1$ in terms of simple Weyl fermions, subject to Lorentz symmetry-breaking perturbation that enormously simplifies the analysis and keeps the outcomes transparent.

In Sec. III A we introduce simple tight-binding models for multi-Weyl semimetals on a cubic lattice. First, we present effective two-band models for such systems with $n=1,2$, and 3 and argue that discrete fourfold rotational $\left(C_{4}\right)$ symmetry protects such higher-order touching of Kramers nondegenerate valence and conduction bands at two Weyl nodes. Therefore, multi-Weyl nodes are symmetry protected. The resulting band structures are shown in Fig. 2. Moreover, we show that multi-Weyl semimetals with $n=2$ and 3 can be constructed by coupling $n$ copies of the lattice model for simple Weyl semimetal (with $n=1$ ) by the $C_{4}$-symmetry-preserving perturbation. The resulting band structures of these two systems, shown in Fig. 3, are identical to the ones obtained from their corresponding two-band models [see Figs. 2(b) and 2(c)], but only at low energies.

The topological equivalence between these two constructions for the multi-Weyl systems is then further substantiated from the bulk-boundary correspondence, encoded through the number of Fermi arc surface states (see Sec. III B). Note that a multi-Weyl semimetal, consisting of an (anti)monopole of charge $n$, supports $n$ copies of the Fermi arcs. Indeed, we find $n$ copies of the Fermi arcs connecting two Weyl nodes of charge $n$ from the two-band models (see Fig. 4). Furthermore, we also observe two and three copies of the arc states for double- and triple-Weyl semimetals, respectively, when they are constructed by coupling two and three copies of simple Weyl fermions by a $C_{4}$-symmetry-preserving perturbation (see Fig. 5).

Upon constructing multi-Weyl semimetals by coupling simple Weyl fermions with a static non-Abelian SU(2) gauge field, we derive the effective field theory of these systems in Sec. IV. The effective field theory for multi-Weyl semimetals displays both Abelian U(1) and SU(2) non-Abelian (only for $n>1$ ) anomalies. To this end, we compute the Ward identities for both covariant and consistent (related by the BardeenZumino polynomials) Abelian and non-Abelian currents. One of our main results is the generalization of the anomalous Hall effect for multi-Weyl semimetals and its non-Abelian variation, captured by, for example, the regular $\left(\rho_{e}\right)$ and isospin $\left(\rho_{3}\right)$ charge densities, respectively, given by

$$
\rho_{e}=n \frac{e^{2}}{2 \pi^{2}}(\mathbf{b} \cdot \mathbf{B}), \quad \rho_{3}=\frac{c(n)}{2 \pi^{2}}\left(\mathbf{b} \cdot \mathbf{B}_{3}\right) .
$$

In the above expressions, $e$ is the electric charge, $2|\mathbf{b}|$ is the separation of left and right Weyl nodes, $\mathbf{B}$ is the external Abelian magnetic field, $\mathbf{B}_{3}=\left(0,0, \Delta^{2}\right)$ is the static nonAbelian magnetic field (present only for $n>1$ ), and $c(n)$ is a coefficient set by the representation of the SU(2) Lie group. Specifically, $c(n)=\frac{1}{2}$ and 2 for $n=2$ and 3 , respectively. We also find that the chiral magnetic effect vanishes for both vector Abelian and non-Abelian currents [see the discussion in Sec. IV B, after Eq. (54)].
To test the validity of the field-theoretic predictions from Sec. IV, we first compute the Abelian or U(1) charge density $\rho_{e}$ in the presence of a static external magnetic field from all the lattice models for multi-Weyl systems, introduced in Sec. III. The methodology is discussed in Sec. V and the results are displayed in Fig. 6. We find that the field-theoretic predictions [see Eq. (1)] show excellent agreement with the scaling of the Abelian charge density with the external magnetic field flux, at least when it is small (cyclotron frequency being much smaller than lattice momenta), irrespective of the microscopic details. As a penultimate topic, we compute the non-Abelian or isospin density $\rho_{3}$, capturing the signature of non-Abelian anomalies [see Eq. (1)], for the multi-Weyl semimetals with $n=2$ and 3 , but only from their four- and six-band lattice models, respectively. The results are shown in Fig. 7, displaying excellent agreement with the field-theoretic predictions.

The topological nature of anomalies in certain cases protects their associated transport, showing universalities even when some symmetries are broken [38,39]. Therefore, the microscopic details of different models become irrelevant, as long as the anomalous structure does not differ between them $[40,41]$. On the other hand, the computation of anomalyinduced transport coefficients with standard quantum field theory techniques can be plagued with subtleties and ambiguities [9-15], which have been solved and understood with the help of the holographic techniques $[42,43]$. Therefore, we address the imprint of the symmetry-breaking parameter $(\Delta)$ in various anomaly-induced transports from a simple toy model for a (strongly) interacting multi-Weyl semimetal using the holographic techniques (see Sec. VI). The particular model we study is consistent with the predictions of the effective field theory and shows a renormalization of the non-Abelian current in the infrared regime, as expected due to the explicit symmetry breaking introduced by $\Delta$. The main outcome from this section is the survival of the non-Abelian transport at low energies, opening a possibility of observing non-Abelian anomaly and the nonrenormalization of the Abelian anomalyinduced transport in multi-Weyl semimetals. Therefore, altogether the current discussion presents a comprehensive study of anomalies in Lorentz symmetry violating multi-Weyl semimetals, which in the future can be extended to address similar issues for multifold fermions $[44,45]$.

\section{B. Outline}

The rest of the paper is organized as follows. In the next section we discuss the low-energy models for multiWeyl semimetals and compute their topological invariant. Section III is devoted to the discussion on the lattice models for these systems on a cubic lattice. In this section we also establish the bulk-boundary correspondence by constructing (numerically) the Fermi arc surface states for multi-Weyl semimetals. The effective field theories, capturing the signature of quantum anomalies, for multi-Weyl semimetals are derived in Sec. IV. The field-theoretic predictions from this section are numerically anchored from the representative tight-binding models in Sec. V. The holographic description and transport coefficients are derived from the gauge-gravity duality in Sec. VI. A discussion of our findings and some 
future directions are highlighted in Sec. VII. Additional technical details are relegated to the Appendixes.

\section{MULTI-WEYL FERMIONS}

We begin the discussion by focusing on the effective lowenergy models for multi-Weyl systems, consisting of a pair of (anti)monopoles of charge $n$ [31-36,46-52], where $n$ is an integer. The Hamiltonian operator describing such a system takes the form

$$
H_{n}^{ \pm}=\alpha_{n} p_{\perp}^{n}\left[\cos \left(n \phi_{p}\right) \tau_{x}+\sin \left(n \phi_{p}\right) \tau_{y}\right] \pm v p_{z} \tau_{z},
$$

where $p_{\perp}=\left(p_{x}^{2}+p_{y}^{2}\right)^{1 / 2}$ and \pm correspond to two valleys acting, respectively, as the monopole (source) and antimonopole (sink) of Abelian Berry curvature. Around these two points low-energy excitations are described in terms of left and right chiral fermions, respectively. Momentum $\mathbf{p}$ is measured from the Weyl node. The set of Pauli matrices $\boldsymbol{\tau}=$ $\left(\tau_{x}, \tau_{y}, \tau_{z}\right)$ operates on the pseudospin indices. The energy spectra in the immediate vicinity of the Weyl nodes take the form $\pm \epsilon_{\mathbf{p}}$, where \pm correspond to the conduction and valence bands, respectively, and

$$
\epsilon_{\mathbf{p}}=\sqrt{\alpha_{n}^{2} p_{\perp}^{2 n}+v^{2} p_{z}^{2}} .
$$

For $n=1$ and 2 the parameter $\alpha_{n}$ bears the dimension of velocity and inverse mass, respectively, while $v$ is the Fermi velocity in the $z$ direction. The energy dispersions along various high-symmetry directions for $n=1,2$, and 3 are shown in Fig. 1.

The topological invariant of Weyl systems is given by the integer (anti)monopole charge, which can be computed in the following way. For concreteness, we now focus near one valley, hosting left chiral fermions, and introduce the coordinate system

$$
\left(p_{x}, p_{y}, p_{z}\right)=\left(p_{\perp} \cos \phi, p_{\perp} \sin \phi, \frac{\epsilon_{\mathbf{p}}}{v} \cos \theta\right),
$$

where $p_{\perp}=\left(\epsilon_{\mathbf{p}} \sin \theta / \alpha_{n}\right)^{1 / n}$. The Berry curvature of the conduction band then takes the form [53]

$$
\boldsymbol{\Omega}_{\mathbf{p}}=\frac{n^{2} \alpha_{n}^{2}}{2 \epsilon_{\mathbf{p}}^{2}}\left(\frac{\epsilon_{\mathbf{p}} \sin \theta}{\alpha_{n}}\right)^{2(n-1) / n} h_{1} \hat{\epsilon},
$$

where $\hat{\epsilon}$ is the unit-norm radial vector and

$$
h_{1}=\frac{1}{v}\left[\cos ^{2} \theta+\frac{v^{2}}{n^{2} \epsilon_{\mathbf{p}}^{2}}\left(\frac{\epsilon_{\mathbf{p}} \sin \theta}{\alpha_{n}}\right)^{2 / n}\right]^{1 / 2} .
$$

The integer monopole charge can then be obtained by integrating the Berry curvature over a unit sphere $(\Sigma)$, defined by $\epsilon_{\mathbf{p}}=1$, around the Weyl node, yielding

$$
\frac{1}{2 \pi} \oint_{\Sigma} \boldsymbol{\Omega}_{\mathbf{p}} \cdot d \mathbf{S}=n
$$

Even though the low-energy model for multi-Weyl semimetals correctly captures the topological invariant of the system, one can construct the Weyl models with $n>1$ by coupling $n$ copies (hereafter referred to as flavor) of simple Weyl fermions in the following way. This construction follows the spirit of realizing higher-order band touching in multilayer graphenelike systems by introducing interlayer tunneling $[33,54,55]$. In addition, this construction opens an efficient route to arrive at the effective field theoretic description for Weyl systems with $n>1$ (see Sec. IV). We focus near the left chiral valley and introduce the Hamiltonian operator

$$
\begin{aligned}
H_{n}^{\text {coup }}= & {\left[v_{\perp}\left(p_{x} \tau_{x}+p_{y} \tau_{y}\right)+v p_{z} \tau_{z}\right] \otimes \mathbb{1}_{n \times n} } \\
& +\Delta\left(\tau_{x} \otimes s_{x}^{n}+\tau_{y} \otimes s_{y}^{n}\right),
\end{aligned}
$$

where $\mathbb{1}_{n \times n}, s_{x}^{n}$, and $s_{y}^{n}$ operate on the flavor index, while the $\boldsymbol{\tau}$ operate on the pseudospin index. Note that the first term in $H_{n}^{\text {coup }}$ corresponds to $n$ decoupled flavors of simple Weyl fermions, while the term proportional to $\Delta$ introduces nontrivial coupling between them. ${ }^{1}$ For any integer $n, \mathbb{1}_{n \times n}$ is an $n$-dimensional identity matrix and $s^{n}$ are the generators of the spin- $(n-1) / 2$ representation of $\mathrm{SU}(2)$. In particular, for $n=2, s^{n}=\sigma / 2$, where $\sigma$ are the Pauli matrices, while for $n=3$,

$$
s_{x}^{n}=\frac{\lambda_{1}+\lambda_{6}}{\sqrt{2}}, \quad s_{y}^{n}=\frac{\lambda_{2}+\lambda_{7}}{\sqrt{2}}, \quad s_{z}^{n}=\frac{\lambda_{3}+\sqrt{3} \lambda_{8}}{2},
$$

and $\lambda$ are the Gell-Mann matrices [56]. In principle, one can generalize this construction for an arbitrary integer value of $n$; however, in a crystalline environment only Weyl nodes with $n \leqslant 3$ are symmetry protected. So we focus here on Weyl systems with $n=1,2$, and 3 . The derivation of the low-energy Hamiltonian [see Eq. (2)] for multi-Weyl semimetals with $n=2$ and 3 , starting from the above coupled models, is shown in Appendix A.

For $n=2$ the energy spectra are composed of four branches, given by $\pm \epsilon_{\mathbf{p}}^{q}$, where

$$
\epsilon_{\mathbf{p}}^{q}=\left[\left(\sqrt{\frac{\Delta^{2}}{4}+v_{\perp}^{2} p_{\perp}^{2}}-(-1)^{q} \frac{\Delta}{2}\right)^{2}+v^{2} p_{z}^{2}\right]^{1 / 2}
$$

for $q=0$ and 1 and \pm correspond to the conduction and valence bands, respectively. Note that only the $q=0$ branch displays band touching at $\mathbf{p}=0$, while the $q=1$ branch is fully gapped for any $\Delta \neq 0$. Expanding $\epsilon_{\mathbf{p}}^{0}$ for large $\Delta$ and small $p_{\perp}$, we obtain

$$
\epsilon_{\mathbf{p}}^{0}=\left[\frac{v_{\perp}^{4} p_{\perp}^{4}}{\Delta^{2}}+v^{2} p_{z}^{2}+O\left(\frac{v_{\perp}^{6} p_{\perp}^{6}}{\Delta^{4}}\right)\right]^{1 / 2},
$$

which agrees with the expression from Eq. (3) to the order $p_{\perp}^{4}$, with $\alpha_{2}=v_{\perp}^{2} / \Delta$, bearing the dimension of inverse mass. Shortly, we show that the pair of split-off bands are topologically trivial, while the band touching point within the $q=0$ sector acts as a monopole of charge $n=2$. On the other hand, for $n=3$, the energy spectra are composed of six branches

\footnotetext{
${ }^{1}$ Note that the form of such interflavor coupling is not unique. One can choose it to be $\Delta\left(\tau_{y} \otimes s_{x}^{n}-\tau_{x} \otimes s_{y}^{n}\right)$, which leaves all the physical outcomes unchanged.
} 


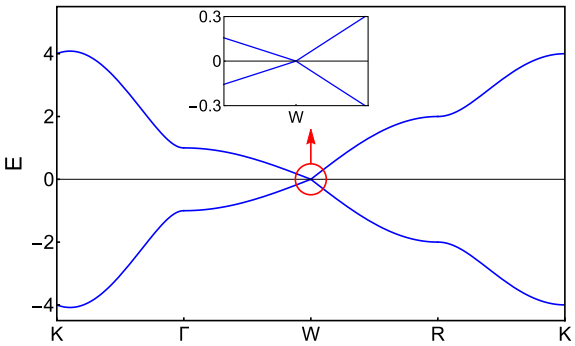

(a)

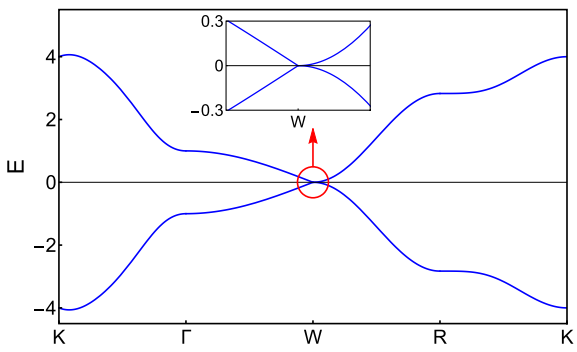

(b)

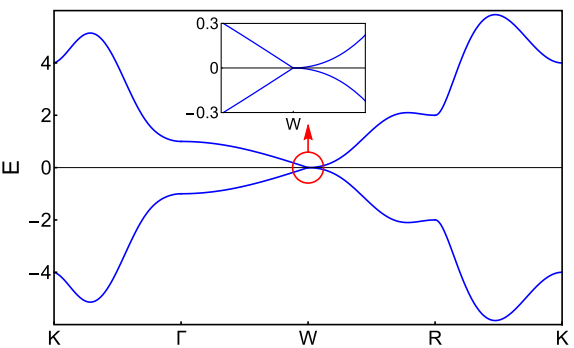

(c)

FIG. 2. Band structure of (a) simple $(n=1)$, (b) double $(n=2)$, and (c) triple $(n=3)$ Weyl semimetals from the corresponding effective two band models [see Eqs. (15)-(18)]. We set $t=t_{0}=t_{z}=1$ in the tight-binding model, and the lattice constant $a=1$. We here take the path $K \rightarrow \Gamma \rightarrow W \rightarrow R \rightarrow K$, where $K, \Gamma, W$, and $R$ stand for $(\pi, \pi, \pi / 2),(0,0,0),(0,0, \pi / 2)$, and $(\pi, 0, \pi / 2)$, respectively. The energy $E$ is measured in units of $t$. Note that Weyl nodes are located at $(0,0, \pm \pi / 2)$, around which dispersion always scales linearly in the $z$ direction. The insets display linear, quadratic, and cubic dispersion in the $\left(k_{x}, k_{y}\right)$ plane, respectively. Due to the absence of particle-hole asymmetry, the Weyl nodes are always pinned at zero energy. Realizations of double- and triple-Weyl systems from four- and six-band models, respectively, are shown in Fig. 3.

$\pm \epsilon_{\mathbf{p}}^{q}$ for $q=0,1,2$, where

$$
\begin{aligned}
\epsilon_{\mathbf{p}}^{q}= & \left\{v_{\perp}^{2} p_{\perp}^{2}+v^{2} p_{z}^{2}+\frac{2 \Delta^{2}}{3}+\frac{2 \Delta}{3} \sqrt{6 v_{\perp}^{2} p_{\perp}^{2}+\Delta^{2}}\right. \\
& \left.\times \cos \left[\frac{1}{3} \cos ^{-1}\left(\frac{9 v_{\perp}^{2} p_{\perp}^{2} \Delta-2 \Delta^{3}}{2\left(6 v_{\perp}^{2} p_{\perp}^{2}+\Delta^{2}\right)^{3 / 2}}\right)-\frac{2 \pi(2-q)}{3}\right]\right\}^{1 / 2} .
\end{aligned}
$$

Note that only the $q=0$ branch displays band touching at $\mathbf{p}=0$, which acts as a monopole of charge $n=3$, while the remaining four bands are completely gapped and topologically trivial. Expanding $\epsilon_{\mathbf{p}}^{0}$ for large $\Delta$ and small $p_{\perp}$, we obtain

$$
\epsilon_{\mathbf{p}}^{0}=\left[\frac{v_{\perp}^{6} p_{\perp}^{6}}{\Delta^{4}}+v^{2} p_{z}^{2}+O\left(\frac{v_{\perp}^{8} p_{\perp}^{8}}{\Delta^{6}}\right)\right]^{1 / 2},
$$

which agrees with Eq. (3) to the order $p_{\perp}^{6}$, with $\alpha_{3}=v_{\perp}^{3} / \Delta^{2}$. The above construction of generating multi-Weyl systems by coupling simple-Weyl fermions, however, is not an artifact of low-energy approximation. In Sec. III we show that such construction is operative even when we start from the lattice regularized models.

Finally, we compute the Berry curvature for each band for multi-Weyl systems with $n>1$, obtained by coupling $n$ flavors of simple Weyl fermions. For $n=2$, we perform this exercise analytically, by introducing the nonorthogonal curvilinear coordinate system

$$
p_{x}=p_{\perp} \cos \phi, \quad p_{y}=p_{\perp} \sin \phi, \quad p_{z}=\epsilon_{\mathbf{p}}^{0} \cos \theta,
$$

where now $p_{\perp}^{2}=\epsilon_{\mathbf{p}}^{0} \sin \theta\left(\epsilon_{\mathbf{p}}^{0} \sin \theta+\Delta\right)$ and $\epsilon_{\mathbf{p}}^{0}$ is displayed in Eq. (9). In this coordinate system, the Berry curvature for the lower conduction band takes a compact form

$$
\begin{aligned}
\boldsymbol{\Omega}= & -\frac{\Delta \sin 2 \theta}{\epsilon_{\mathbf{p}}^{0}\left(\Delta+2 \epsilon_{\mathbf{p}}^{0} \sin \theta\right)^{3}} \mathbf{e}_{\theta} \\
& +\frac{2 \Delta \epsilon_{\mathbf{p}}^{0}+2 \sin \theta\left[\Delta^{2}+2 \epsilon_{\mathbf{p}}^{0} \Delta \sin \theta+2\left(\epsilon_{\mathbf{p}}^{0}\right)^{2} \sin ^{2} \theta\right]}{\epsilon_{\mathbf{p}}^{0}\left(\Delta+2 \epsilon_{\mathbf{p}}^{0} \sin \theta\right)^{3}} \mathbf{e}_{\epsilon},
\end{aligned}
$$

where $\mathbf{e}_{\epsilon}=\partial \mathbf{r} / \partial \epsilon_{\mathbf{p}}^{0}$ and $\mathbf{e}_{\theta}=\partial \mathbf{r} / \partial \theta$ are the covariant basis vectors. From the above expression for the Berry curvature, we can immediately compute the integer charge associated with the band touching point from Eq. (7), yielding $n=2$ for any $\Delta \neq 0$. The expression for the Berry curvature for the gapped valence and conduction bands is quite lengthy and not very instructive. However, when we integrate the Berry curvature over a closed surface [see Eq. (7)], it yields a trivial answer. Therefore, in the four-band construction for doubleWeyl fermions, only the two bands touching each other are topologically nontrivial. For triple-Weyl fermions four gapped bands are topologically trivial, while the monopole charge of the band touching points, where valence and conduction bands meet, is $n=3$.

\section{LATTICE MODEL, BULK-BOUNDARY CORRESPONDENCE, AND FERMI ARCS}

In this section we introduce effective tight-binding models of a cubic lattice yielding multi-Weyl semimetals, possessing only two Weyl nodes. Subsequently, we establish the bulkboundary correspondence for these systems by computing the Fermi arc surface states. These analyses substantiate our discussion from the preceding section. In addition, we also subscribe to these lattice models to test the predictions from the effective field theory for multi-Weyl semimetals (see Sec. IV), discussed in Sec. V.

\section{A. Lattice models}

The lattice model for general Weyl fermions can compactly be written as

$$
H_{\mathrm{Weyl}}=\sum_{\mathbf{k}} \Psi_{\mathbf{k}}^{\dagger}[\mathbf{N}(\mathbf{k}) \cdot \boldsymbol{\tau}] \Psi_{\mathbf{k}},
$$

where $\Psi_{\mathbf{k}}^{\top}=\left(c_{\mathbf{k}, \uparrow}, c_{\mathbf{k}, \downarrow}\right)$ is a two-component spinor, with $c_{\mathbf{k}, \tau}$ the fermion annihilation operator with momentum $\mathbf{k}$ and pseudospin projection $\tau=\uparrow, \downarrow$. The effective two-band theory emerging from the above tight-binding model gives rise to left and right chiral Weyl fermions near $\left(0,0, \pm \frac{\pi}{2 a}\right)$, respectively, 
if we choose

$$
N_{3}(\mathbf{k})=t_{z} \cos \left(k_{z} a\right)+t_{0}\left[2-\cos \left(k_{x} a\right)-\cos \left(k_{y} a\right)\right] .
$$

For convenience, we set the lattice spacing $a$ to be unity. Then simple, double-, and triple-Weyl fermions are realized when we set [57-59]

$$
N_{x}(\mathbf{k})=t \times \begin{cases}\sin \left(k_{x}\right) & \text { for } n=1 \\ \cos \left(k_{x}\right)-\cos \left(k_{y}\right) & \text { for } n=2 \\ \sin \left(k_{x}\right)\left[3 \cos \left(k_{y}\right)-\cos \left(k_{x}\right)-2\right] & \text { for } n=3\end{cases}
$$

and

$$
N_{y}(\mathbf{k})=t \times \begin{cases}\sin \left(k_{y}\right) & \text { for } n=1 \\ \sin \left(k_{x}\right) \sin \left(k_{y}\right) & \text { for } n=2 \\ \sin \left(k_{y}\right)\left[3 \cos \left(k_{x}\right)-\cos \left(k_{y}\right)-2\right] & \text { for } n=3 .\end{cases}
$$

The resulting band structures for $n=1,2$, and 3 are shown in Fig. 2. Notice that the above tight-binding models produce only a pair of Weyl nodes at $(0,0, \pm \pi / 2)$, around which the effective low-energy models assume the form announced in Sec. II [see Eq. (2)].

The band touching points in multi-Weyl semimetals are protected by the four-four or $C_{4}$ rotation about the $z$ axis, a bona fide symmetry operation of the $D_{4 d}$ point group. Under such a $C_{4}$ rotation $\left(k_{x}, k_{y}, k_{z}\right) \rightarrow\left(-k_{y}, k_{x}, k_{z}\right)$. When such rotation in the momentum space is accompanied by a rotation by an angle $\theta_{\mathrm{SP}}^{n}=n \frac{\pi}{2}$ in the pseudospin space, captured by the unitary operator $\mathcal{R}_{\mathrm{SP}}\left(n \frac{\pi}{2}\right)=\exp \left(i \theta_{\mathrm{SP}}^{n} \tau_{z}\right)$, the Hamiltonian operator for $n=1$ and 2 remains completely invariant. The situation for $n=3$ is slightly more subtle, as $N_{x, y}(\mathbf{k}) \rightarrow$ $-N_{x, y}(\mathbf{k})$. Nonetheless, the monopole and antimonopole map onto themselves under such $C_{4}$ rotations, leaving the tripleWeyl points symmetry protected. On the other hand, if we take $N_{x}(\mathbf{k}) \leftrightarrow N_{y}(\mathbf{k})$ for $n=3$, all the outcomes remain unchanged, but the corresponding Hamiltonian operator remains completely invariant under the $C_{4}$ rotation. Hence, multi-Weyl points are symmetry protected in a system possessing a $D_{4 d}$ symmetry.

The multi-Weyl semimetals with $n>1$ can also be realized by properly coupling $n$ copies of simple Weyl semimetals. We discussed this construction from the continuum or lowenergy models in Sec. II. We now test the validity of such a construction, starting from the lattice models for $n=1$ Weyl fermions, given by

$$
\begin{aligned}
H_{\mathrm{SW}}= & t\left[\sin \left(k_{x}\right) \tau_{x}+\sin \left(k_{y}\right) \tau_{y}\right]+\left\{t_{z} \cos \left(k_{z}\right)\right. \\
& \left.+t_{0}\left[2-\cos \left(k_{x}\right)-\cos \left(k_{y}\right)\right]\right\} \tau_{z} .
\end{aligned}
$$

Following Eq. (8), we construct the lattice model for multiWeyl semimetals by coupling $n$ copies of simple Weyl semimetals according to

$$
H_{n, \text { latt }}^{\text {coup }}=H_{\mathrm{SW}} \otimes \mathbb{1}_{n \times n}+\Delta\left(\tau_{x} \otimes s_{x}^{n}+\tau_{y} \otimes s_{y}^{n}\right) .
$$

The notation is the same as in Sec. II. The resulting band structures for $n=2$ and 3 are shown in Fig. 3. For $n=2$ and 3, two and four bands are completely gapped, respectively, while the two remaining bands touch each other at $(0,0, \pm \pi / 2)$. The energy dispersions around these points are quadratic and cubic, respectively, with the in-plane components of momenta, but always scale linearly with their $z$ component.

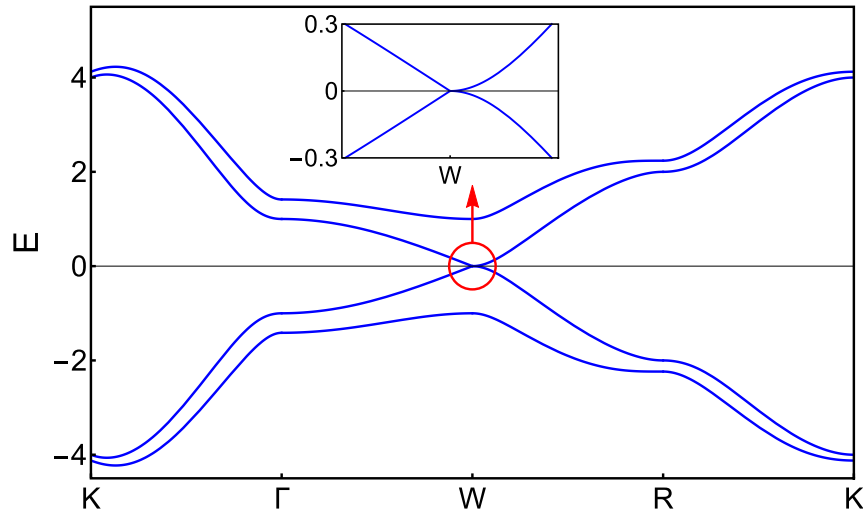

(a)

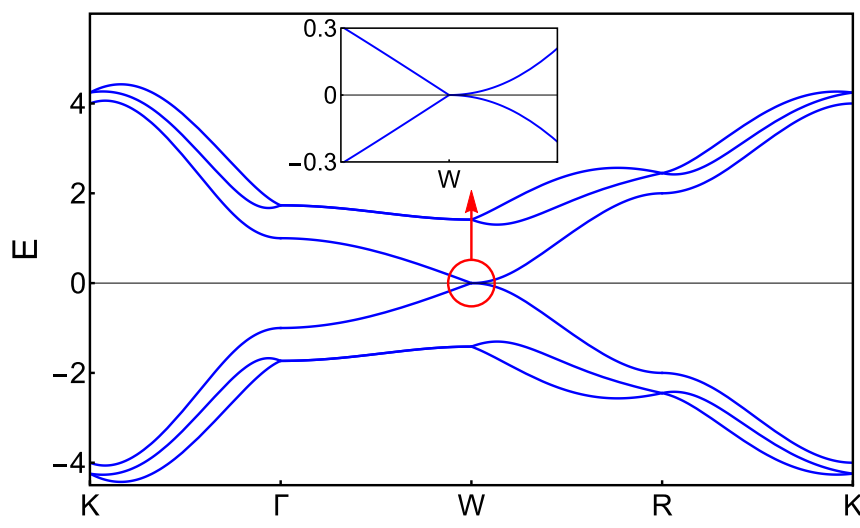

(b)

FIG. 3. Band structure of (a) double- and (b) triple-Weyl semimetals from four- and six-band models, respectively [see Eqs. (19) and (20)]. For numerical diagonalization we set $t=t_{0}=$ $t_{z}=\Delta=1$ in the tight-binding models, and the lattice spacing $a=$ 1. Note that these models also display quadratic [see (a)] and cubic [see (b)] dispersion in the $\left(k_{x}, k_{y}\right)$ plane around the Weyl nodes located at $W=(0,0, \pm \pi / 2)$ [compare with Figs. 2(b) and 2(c)]. The dispersion always scales linearly with $k_{z}$.

\section{B. Fermi arcs}

Previously, in Sec. II, we showed that the four- and six-band models for double- and triple-Weyl fermions, respectively [see Eq. (8)], and their low-energy description in terms of the two-band models [see Eq. (2)] yield identical topological invariants (the monopole charge). The monopole charge determines the integer topological invariant of the system that in turn also dictates the number of topologically protected Fermi arc surface states, connecting two Weyl nodes of opposite chiralities. Therefore, equivalence between the four- (six-) band model for the double- (triple-) Weyl fermions [see Eq. (20)] and their two-band models [see Eqs. (15)-(18)] can be established by comparing the number of Fermi arcs for multi-Weyl systems from these two sets of tight-binding models. The results are shown in Figs. 4 and 5.

To compute the Fermi arc surface states we impose periodic boundaries in the $y$ and $z$ directions such that $k_{y}$ and $k_{z}$ can be treated as good quantum numbers. However, we implement an open boundary in the $x$ direction, along which 


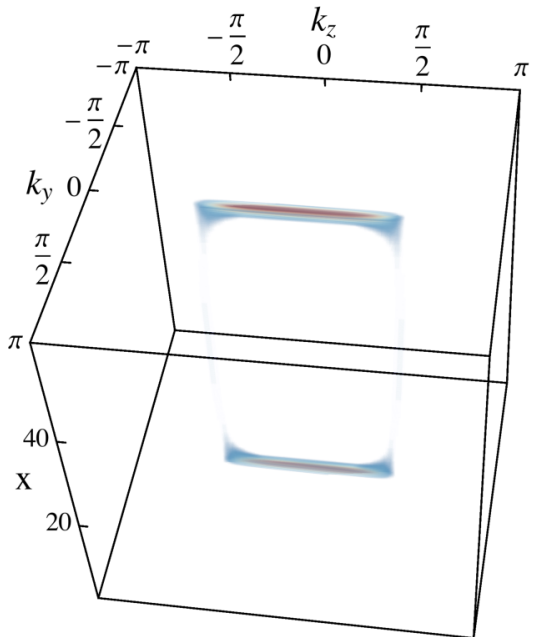

(a)

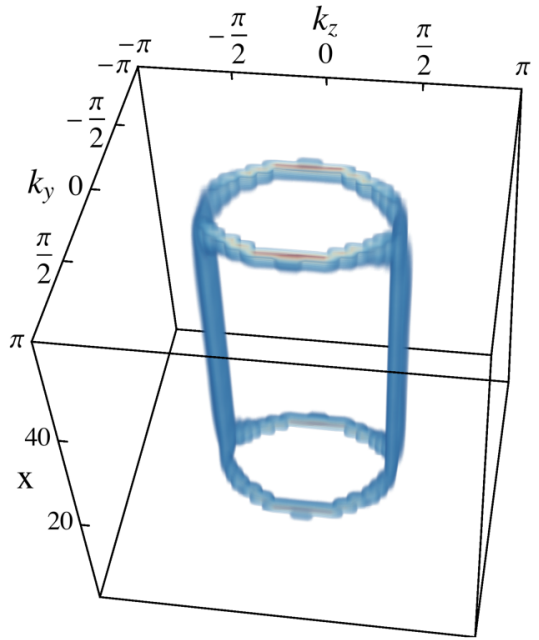

(b)

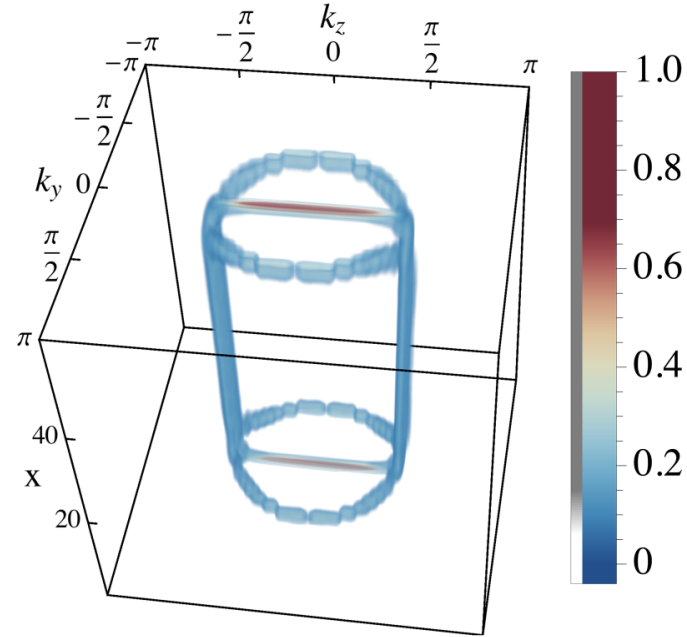

(c)

FIG. 4. Topologically protected Fermi arc surface states for (a) simple $(n=1)$, (b) double- $(n=2)$, and (c) triple- $(n=3)$ Weyl semimetals, obtained from their two-band models [see Eqs. (15)-(18)]. For numerical diagonalization we implement a mixed momentum (along $k_{y}$ and $k_{z}$ ) and real-space or Wannier (along $x$ ) representation. The linear dimensionality of the system along $x$ is $L=60$ (see Sec. III B for details). Consequently, the Fermi arc surface states are localized on the top and bottom surfaces. We set $t=t_{z}=t_{0}=1$. We display here the square of the amplitude of the low-energy states within an energy window $\Delta E=0.1$ for $n=1, \Delta E=0.08$ for $n=2$, and $\Delta E=0.05$ for $n=3$ around zero energy. The number of Fermi arcs is equal to $n$, anchoring the bulk-boundary correspondence for multi-Weyl semimetals. In addition, the Fermi arcs from opposite surfaces (top and bottom) get connected through the bulk Weyl points (acting as defects, namely, monopole and antimonopole, in the momentum space), where the band gap vanishes.

the linear dimensionality of the system is denoted by $L$ [60,61]. In such a mixed Bloch-Wannier representation, the Fermi arcs are localized on the top and bottom surfaces, as shown in Figs. 4 and 5. Specifically, in Fig. 4 we show the topologically protected Fermi arcs for multi-Weyl semimetals, constructed from their two-band tight-binding models [see Eqs. (15)-(18)]. We find that a multi-Weyl semimetal, characterized by integer (anti)monopole charge $n$, supports exactly $n$ copies of Fermi arc surface states. This observation establishes the bulk-boundary correspondence for this family of gapless topological semimetals. On the other hand, in Fig. 5 we show the Fermi arcs for double- and triple-Weyl semimetals, but constructed from the four- and six-band models [see Eqs. (19) and (20)], respectively. Once again we find that these two systems host, respectively, two and three copies of the Fermi arcs on the top and bottom surfaces. This outcome, besides supporting the bulk-boundary correspondence, also anchors the topological equivalence between the multiband and two-band representations for the double- and triple-Weyl semimetals on a lattice. To appreciate some additional salient features of the arc states, next we consider their microscopic origin.

Any general Weyl semimetal hosting an (anti)monopole of charge $n$ can be constructed by stacking two-dimensional layers of quantum anomalous Hall insulators, occupying the $x y$ plane, in the momentum space along the $k_{z}$ direction within the range $-K_{0} \leqslant k_{z} \leqslant K_{0}$, where $K_{0}=\frac{\pi}{2}$ in our lattice construction. The first Chern number of each such anomalous Hall insulator is $n$ and it supports $n$ copies of one-dimensional chiral edge modes, with $n$ states at precisely zero energy. The collection of such zero-energy states within the range $-K_{0} \leqslant k_{z} \leqslant K_{0}$ constitutes $n$ copies of the Fermi arc surface states, shown in Figs. 4 and 5. Also note that the localization length of each zero-energy mode is inversely proportional to the bulk gap of the underlying two-dimensional anomalous Hall insulator for a given $k_{z}$. In our lattice models, such a gap is largest when $k_{z}=0$ and it vanishes at $k_{z}= \pm \frac{\pi}{2}$. Otherwise, this gap decreases smoothly as we approach $k_{z}=$ $\pm \frac{\pi}{2}$ from the center of the surface Brillouin zone $\left(k_{z}=0\right)$. Consequently, the surface localization of each copy of Fermi arcs decreases monotonically as we approach two Weyl points from the center of the arcs. Ultimately, at $k_{z}= \pm \frac{\pi}{2}$ the arcs are completely delocalized, and at these two points arcs from the top and bottom surfaces get connected via the bulk Weyl nodes. This feature can be seen from Figs. 4 and 5. Next we proceed to derive the effective field theory of these systems.

\section{EFFECTIVE FIELD THEORY}

All global symmetries, present in a classical action, do not necessarily survive after quantization [1]. Possibly the best known examples of this phenomenon are the ones related to the chiral anomalies. In particular, the axial anomaly is responsible for the celebrated decay of the pion into two photons [2,3]. Furthermore, it also leaves signatures on anomalyinduced transports that have recently attracted ample attention in the context of Dirac and Weyl semimetals in condensedmatter systems, quark-gluon plasma in heavy-ion colliders, and magnetized plasmas in cosmology, for example. In this section we derive the effective field theory for multi-Weyl semimetals, reveal the anomaly structure therein, and discuss the imprints of the anomaly structure on various transports.

After establishing the Hamiltonian description for multiWeyl semimetals, we seek to formulate the corresponding Lagrangian formalism, which allows us to derive the effective field theory for these systems. Performing a Legendre 


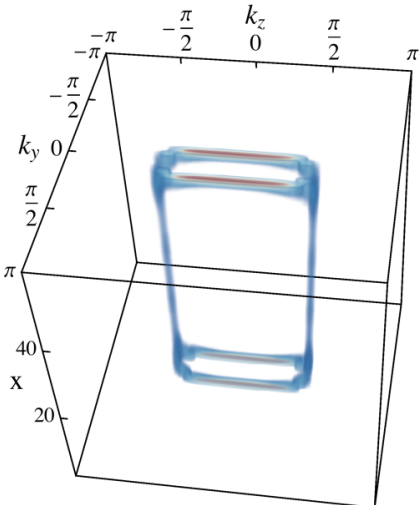

(a)

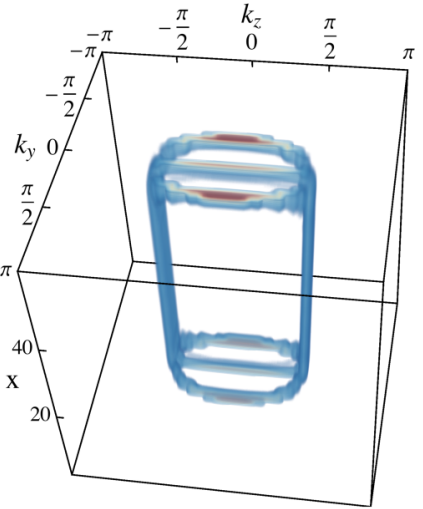

(b)
FIG. 5. Fermi arc surface states for (a) double- $(n=2)$ and (b) triple- $(n=3)$ Weyl semimetals, obtained, respectively, from four- and six-band models [see Eqs. (19) and (20)]. For numerical diagonalization we follow the same approach, mentioned in the caption of Fig. 4, and set $t=t_{z}=t_{0}=\Delta=1$ and $a=1$. We display here the square of the amplitude of the low-energy states within an energy window $\Delta E=0.05$ for $n=2$ and $\Delta E=0.04$ for $n=3$. Note that double- and triple-Weyl semimetals support, respectively, two and three copies of Fermi arc surface states, as we obtained from their two-band representations [see Figs. 4(b) and 4(c)]. This observation establishes the topological equivalence among these models and the bulk-boundary correspondence in these gapless topological systems. The color scale is the same as in Fig. 4.

transformation on the continuum Hamiltonian [see Eq. (8)], we obtain the Lagrangian for left chiral fermions $\left(\psi_{L}\right)$

$$
\mathcal{L}_{L}=i \psi_{L}^{\dagger} \tau^{\mu}\left[\partial_{\mu}-i \Delta\left(\delta_{\mu}^{x} s_{x}+\delta_{\mu}^{y} s_{y}\right)\right] \psi_{L},
$$

where $\tau^{\mu}=(1, \vec{\tau})$. Einstein's summation convention over repeated indices is assumed throughout. The above expression allows us to construct a generalized formalism for multiWeyl semimetals with a non-Abelian $\mathrm{U}(2)_{L}$ flavor symmetry, in the presence of a non-Abelian background gauge field $A_{\mu}^{a} s_{a}=\mathcal{A}_{\mu}^{0} s_{0}+\mathbb{A}_{\mu}^{i} s_{i}$ according to

$$
\mathcal{L}_{L}=i \psi_{L}^{\dagger} \tau^{\mu}\left[\partial_{\mu}-i A_{\mu}^{a} s_{a}\right] \psi_{L},
$$

where $s_{a}=\left(s_{0}, s_{i}\right)$ with $i=x, y, z$ are the generators of $\mathrm{U}(1)_{L} \times \mathrm{SU}(2)_{L}$. In particular, the static background field giving rise to nonlinear dispersion (in the $x y$ plane) in multiWeyl systems can be written as

$$
A_{\mu}^{a}=\Delta\left(\delta_{\mu}^{x} \delta^{a x}+\delta_{\mu}^{y} \delta^{a y}\right) .
$$

From now on, we denote the field strength associated with $A_{\mu}$ by $F_{\mu \nu}$, the Abelian gauge field by $\mathcal{F}_{\mu \nu}$, and the SU(2) gauge field by $G_{\mu \nu}$. Note that the non-Abelian field, giving rise to the multi-Weyl semimetals, picks a preferred direction and reduces the initial $\mathrm{SO}(3,1) \times \mathrm{SU}(2)_{L}$ symmetry group of $n$ decoupled copies of simple Weyl fermions to the diagonal $\mathrm{SO}(1,1) \times \mathrm{U}(1)_{3_{L}}$ symmetry.

Let us assume that we have a theory for left chiral fermions, transforming in some representation $\mathcal{R}(\mathcal{G})$ of the Lie group $\mathcal{G}$. The corresponding generators $s_{a}$ of the Lie algebra satisfy

$$
\left[s_{a}, s_{b}\right]=i f^{a b c} s_{c},
$$

where $f^{a b c}$ is the structure factor of the Lie group $\mathcal{G}$. For theories with such a flavor symmetry, the associated anomalous currents $J_{a}^{\mu}$ satisfy the Ward identities in their covariant form

$$
\begin{gathered}
\mathcal{D}_{\mu} J_{a}^{\mu}=\frac{d_{a b c}}{32 \pi^{2}} \epsilon^{\mu \nu \rho \lambda} F_{\mu \nu}^{b} F_{\rho \lambda}^{c}+\frac{b_{a}}{768 \pi^{2}} \epsilon^{\mu \nu \rho \lambda} R_{\beta \mu \nu}^{\alpha} R_{\alpha \rho \lambda}^{\beta}, \\
\nabla_{\mu} T_{\nu}^{\mu}=F_{\nu \mu}^{a} J_{a}^{\mu}+\frac{b_{a}}{384 \pi^{2}} \mathcal{D}^{\mu}\left(\epsilon^{\sigma \kappa \rho \lambda} F_{\kappa \sigma}^{a} R_{\nu \mu \rho \lambda}\right),
\end{gathered}
$$

where $\mathcal{D}_{\mu}$ is the covariant derivative containing the gauge and metric connections, $\nabla_{\mu}$ is the curved space covariant derivative, $R_{v \mu \rho \lambda}$ is the Riemann curvature tensor, $T^{\mu \nu}$ is the stress-energy tensor, and the anomalous coefficients are $d_{a b c}=\frac{1}{2} \operatorname{Tr}\left[\left\{s_{a}, s_{b}\right\} s_{c}\right]$ and $b_{a}=\operatorname{Tr}\left[s_{a}\right][1,43] .^{2}$ The currents $J_{a}^{\mu}$ cannot be obtained by varying an action with respect to the background fields $A_{\mu}^{a}$ and normally they are called covariant currents. Nonetheless, there exist the consistent currents $\tilde{J}_{a}^{\mu}$ related to $J_{a}^{\mu}$ by the addition of a Chern-Simons polynomial. The consistent currents can be defined as a functional derivative of the action with respect to the background field according to $\tilde{J}_{a}^{\mu}=\delta W / \delta A_{\mu}^{a}$, where $J_{a}^{\mu}=\tilde{J}_{a}^{\mu}+K_{a}^{\mu}$, with

$$
K_{a}^{\mu}=-\frac{1}{48 \pi^{2}} \epsilon^{\mu \nu \rho \lambda} \operatorname{Tr}\left[s_{a}\left(\left\{A_{\nu}, F_{\rho \lambda}\right\}-A_{\nu} A_{\rho} A_{\lambda}\right)\right] .
$$

Thus, the consistent Ward identity reads

$$
\begin{aligned}
\mathcal{D}_{\mu} \tilde{J}_{a}^{\mu}= & \frac{1}{24 \pi^{2}} \epsilon^{\mu \nu \rho \sigma} \operatorname{Tr}\left[s_{a} \partial_{\mu}\left(A_{\nu} \partial_{\rho} A_{\sigma}+\frac{1}{2} A_{\nu} A_{\rho} A_{\sigma}\right)\right] \\
& +\frac{b_{a}}{768 \pi^{2}} \epsilon^{\mu \nu \rho \lambda} R_{\beta \mu \nu}^{\alpha} R_{\alpha \rho \lambda}^{\beta} .
\end{aligned}
$$

To illustrate the applicability of the general theoretical framework discussed so far, we now focus on a theory with one copy of left- and right-handed fermions, coupled to Abelian gauge fields. The Ward identities for the consistent current $\tilde{J}_{e}^{\mu}=\tilde{J}_{L}^{\mu}+\tilde{J}_{R}^{\mu}$ that couples to the gauge field $\mathcal{A}_{\mu}=$ $\frac{1}{2}\left(\mathcal{A}_{\mu}^{L}+\mathcal{A}_{\mu}^{R}\right)$ and the axial current, defined as $\tilde{J}_{5}^{\mu}=\tilde{J}_{L}^{\mu}-\tilde{J}_{R}^{\mu}$, read, respectively,

$$
\begin{aligned}
\nabla_{\mu} \tilde{J}_{e}^{\mu}= & \frac{1}{32 \pi^{2}} \epsilon^{\mu \nu \rho \lambda} \mathcal{F}_{\mu \nu} \mathcal{F}_{\rho \lambda}^{5}, \\
\nabla_{\mu} \tilde{J}_{5}^{\mu}= & \frac{1}{48 \pi^{2}} \epsilon^{\mu \nu \rho \lambda}\left(\mathcal{F}_{\mu \nu} \mathcal{F}_{\rho \lambda}+\mathcal{F}_{\mu \nu}^{5} \mathcal{F}_{\rho \lambda}^{5}\right) \\
& +\frac{1}{768 \pi^{2}} \epsilon^{\mu \nu \rho \lambda} R_{\beta \mu \nu}^{\alpha} R_{\alpha \rho \lambda}^{\beta} .
\end{aligned}
$$

The conservation of electric charge requires that the combination $\mathrm{U}(1)_{L}+\mathrm{U}(1)_{R} \equiv \mathrm{U}(1)_{e}$ should be conserved. However, in Eq. (29) the vector current $\tilde{J}_{e}^{\mu}$ is not conserved. ${ }^{3}$ Nonetheless, this issue can be resolved by noting that the theory is not gauge invariant. Hence, one can add a counterterm, known as

\footnotetext{
${ }^{2}$ For right chiral fermions $d_{a b c}=-\frac{1}{2} \operatorname{Tr}\left[\left\{s_{a}, s_{b}\right\} s_{c}\right]$ and $b_{a}=-\operatorname{Tr}\left[s_{a}\right]$.

${ }^{3}$ Notice that Eq. (29) is an operator equation. Therefore, even though at the fundamental level axial gauge fields do not exist, the three-point function $\left\langle\partial_{\mu} \tilde{J}_{e}^{\mu} \tilde{J}_{e}^{v} \tilde{J}_{5}^{\rho}\right\rangle \neq 0$ spoils the consistency of the theory.
} 
the Bardeen counterterm

$$
W_{\mathrm{BCT}}=-\frac{1}{12 \pi^{2}} \int d^{4} x \epsilon^{\mu \nu \rho \lambda} A_{\mu}^{e} A_{v}^{5} F_{\rho \lambda},
$$

to the original action, which reestablishes the $\mathrm{U}(1)_{e}$ gauge invariance. After introducing this local polynomial, the Ward identities for the newly defined consistent currents

$$
j_{e}=\tilde{J}_{e}+\frac{\delta W_{\mathrm{BCT}}}{\delta A_{\mu}^{e}}, \quad j_{5}=\tilde{J}_{5}+\frac{\delta W_{\mathrm{BCT}}}{\delta A_{\mu}^{5}}
$$

read, respectively,

$$
\begin{aligned}
\nabla_{\mu} j_{e}^{\mu}= & 0, \\
\nabla_{\mu} j_{5}^{\mu}= & \frac{1}{16 \pi^{2}} \epsilon^{\mu \nu \rho \lambda}\left(\mathcal{F}_{\mu \nu} \mathcal{F}_{\rho \lambda}+\frac{1}{3} \mathcal{F}_{\mu \nu}^{5} \mathcal{F}_{\rho \lambda}^{5}\right) \\
& +\frac{1}{768 \pi^{2}} \epsilon^{\mu \nu \rho \lambda} R^{\alpha}{ }_{\beta \mu \nu} R^{\beta}{ }_{\alpha \rho \lambda} .
\end{aligned}
$$

Only after such a redefinition, the current $j_{e}^{\mu}$ associated with the $\mathrm{U}(1)_{e}$ electric charge is conserved, while the axial current $j_{5}^{\mu}$ remains anomalous. The above construction has a natural generalization to the theories with non-Abelian anomalies, which we discuss next.

\section{A. Theory with $U(1) \times S U(2)$ flavor symmetries}

Now we consider multi-Weyl semimetals, in which the left- and right-handed fermions transform under an $\mathrm{SU}(2)_{L / R}$ representation. In this case, the (covariant) anomalous Ward identities [see Eq. (25)] read

$$
\begin{aligned}
\nabla_{\mu} J_{e}^{\mu}= & \frac{n}{8 \pi^{2}} \epsilon^{\mu \nu \rho \lambda} \mathcal{F}_{\mu \nu} \mathcal{F}_{\rho \lambda}^{5}+\frac{c(n)}{8 \pi^{2}} \epsilon^{\mu \nu \rho \lambda} G_{\mu \nu}^{i} G_{\rho \lambda}^{5 i}, \\
\nabla_{\mu} J_{5}^{\mu}= & \frac{n}{16 \pi^{2}} \epsilon^{\mu \nu \rho \lambda}\left(\mathcal{F}_{\mu \nu} \mathcal{F}_{\rho \lambda}+\mathcal{F}_{\mu \nu}^{5} \mathcal{F}_{\rho \lambda}^{5}\right) \\
& +\frac{c(n)}{16 \pi^{2}} \epsilon^{\mu \nu \rho \lambda}\left(G_{\mu \nu}^{i} G_{\rho \lambda}^{i}+G_{\mu \nu}^{5 i} G_{\rho \lambda}^{5 i}\right) \\
& +\frac{n}{384 \pi^{2}} \epsilon^{\mu \nu \rho \lambda} R_{\beta \mu \nu}^{\alpha} R_{\alpha \rho \lambda}^{\beta}, \\
\mathcal{D}_{\mu} J_{i}^{\mu}= & \frac{c(n)}{8 \pi^{2}} \epsilon^{\mu \nu \rho \lambda}\left(\mathcal{F}_{\mu \nu} G_{\rho \lambda}^{5 i}+\mathcal{F}_{\mu \nu}^{5} G_{\rho \lambda}^{i}\right), \\
\mathcal{D}_{\mu} J_{i, 5}^{\mu}= & \frac{c(n)}{8 \pi^{2}} \epsilon^{\mu \nu \rho \lambda}\left(\mathcal{F}_{\mu \nu} G_{\rho \lambda}^{i}+\mathcal{F}_{\mu \nu}^{5} G_{\rho \lambda}^{5 i}\right),
\end{aligned}
$$

where $c(n)$ is defined via the relation $\operatorname{Tr}\left(s_{i} s_{j}\right)=c(n) \delta_{i j}$ for $n>1$. For our choices of the generators, $c(2)=\frac{1}{2}$ and $c(3)=2$. As discussed in the preceding section, the covariant current cannot be obtained by differentiating any functional of the gauge fields. Therefore, they do not couple to the gauge fields. However, Bardeen computed the proper counterterm to construct conserved vector consistent currents [7]. ${ }^{4} \mathrm{Com}-$ bining the Bardeen counterterms with the Bardeen-Zumino polynomial [see Eq. (27)] [29,62], we can write the ChernSimons current, relating the covariant and consistent currents

\footnotetext{
${ }^{4}$ We impose the conservation of the vector non-Abelian current because the continuum version of the lattice $C_{4}$ symmetry corresponds to $\mathrm{U}(1)_{3_{e}}=\mathrm{U}(1)_{3_{L}}+\mathrm{U}(1)_{3_{R}}$, discussed in the preceding section.
}

according to

$$
J_{a}^{\mu}=j_{a}^{\mu}+P_{a}^{\mu},
$$

where

$$
\begin{gathered}
P_{a}^{\mu}=\frac{1}{8 \pi^{2}} \epsilon^{\mu \nu \rho \lambda} \operatorname{Tr}\left[s_{a}\left(A_{v}^{5} F_{\rho \lambda}+F_{\rho \lambda} A_{v}^{5}+\frac{8}{3} i A_{v}^{5} A_{\rho}^{5} A_{\lambda}^{5}\right)\right], \\
P_{a, 5}^{\mu}=\frac{1}{24 \pi^{2}} \epsilon^{\mu \nu \rho \lambda} \operatorname{Tr}\left[s_{a}\left(A_{v}^{5} F_{\rho \lambda}^{5}+F_{\rho \lambda}^{5} A_{v}^{5}\right)\right] .
\end{gathered}
$$

Having understood the anomalous structure of the effective field theory, we now extract the anomaly-induced transport coefficients for multi-Weyl systems.

\section{B. Anomaly-induced transport}

Chiral fermions exhibit nondissipative transport at finite temperature $T$ and density $\mu$, which are intimately related to the chiral anomalies discussed in the previous sections. In particular, the covariant currents within the linear-response approximation were computed in Refs. [40,41,63-69] and read ${ }^{5}$

$$
\begin{gathered}
J_{a}^{\mu}=\sigma_{a b}^{B} B_{b}^{\mu}+\sigma_{a}^{V} \omega^{\mu}, \\
T^{\mu \nu}=\sigma_{a}^{\epsilon, B} u^{(\mu} B_{a}^{\nu)}+\sigma^{\epsilon, V} u^{(\mu} \omega^{\nu)},
\end{gathered}
$$

where $A^{(\mu} C^{\nu)}=\left(A^{\mu} C^{\nu}+A^{\nu} C^{\mu}\right) / 2$. The magnetic and vorticity fields are defined, respectively, as

$$
B_{a}^{\nu}=\epsilon^{\mu \nu \rho \lambda} u_{\nu} \nabla_{\rho} A_{\lambda}^{a}, \quad \omega^{\mu}=\epsilon^{\mu \nu \rho \lambda} u_{\nu} \nabla_{\rho} u_{\lambda}
$$

and $u^{\mu}$ is a unit-norm timelike vector. The nondissipative currents give rise to (a) chiral magnetic conductivities $\left(\sigma_{a b}^{B}\right.$ and $\left.\sigma_{a}^{\epsilon, B}\right)$ and (b) chiral vortical conductivities $\left(\sigma_{a}^{V}\right.$ and $\left.\sigma^{\epsilon, V}\right)$. In the absence of dynamical gauge fields, these quantities are universal and solely determined by the anomaly. In addition, they are given by

$$
\begin{aligned}
\sigma_{a b}^{B} & =\frac{1}{4 \pi^{2}} d_{a b c} \mu^{c}, \\
\sigma_{a}^{V} & =\sigma_{a}^{\epsilon, B}=\frac{1}{8 \pi^{2}} d_{a b c} \mu^{b} \mu^{c}+\frac{T^{2}}{24} b_{a}, \\
\sigma^{\epsilon, V} & =\frac{1}{12 \pi^{2}} d_{a b c} \mu^{a} \mu^{b} \mu^{c}+\frac{T^{2}}{12} b_{a} \mu^{a},
\end{aligned}
$$

where $\mu^{a}=\left(\mu, \mu^{i}\right)$, with $\mu$ and $\mu^{i}$ denoting the regular and flavor chemical potentials, respectively.

For a theory with $\mathrm{U}(1)_{e} \times \mathrm{U}(1)_{5}$ symmetry (describing a simple Weyl semimetal with $n=1$ ), the vector and axial covariant currents are given, respectively, by

$$
\begin{gathered}
\mathbf{J}_{e}=\frac{\mu_{5}}{2 \pi^{2}} \mathbf{B}+\frac{\mu}{2 \pi^{2}} \mathbf{B}_{5}+\frac{\mu \mu_{5}}{2 \pi^{2}} \boldsymbol{\omega} \\
\mathbf{J}_{5}=\frac{\mu}{2 \pi^{2}} \mathbf{B}+\frac{\mu_{5}}{2 \pi^{2}} \mathbf{B}_{5}+\left(\frac{\mu^{2}+\mu_{5}^{2}}{4 \pi^{2}}+\frac{T^{2}}{12}\right) \boldsymbol{\omega} .
\end{gathered}
$$

\footnotetext{
${ }^{5}$ These expressions assume linear response; therefore, in the definition of the non-Abelian magnetic fields, only the linear terms in the gauge fields are considered.
} 
On the other hand, the covariant current arising from the energy-momentum tensor, $J_{\epsilon}^{i}=T^{i 0}$, is

$$
\begin{aligned}
\mathbf{J}_{\epsilon}= & \frac{\mu \mu_{5}}{2 \pi^{2}} \mathbf{B}+\left(\frac{\mu^{2}+\mu_{5}^{2}}{4 \pi^{2}}+\frac{T^{2}}{12}\right) \mathbf{B}_{5} \\
& +\left(\frac{\mu_{5}}{6 \pi^{2}}\left(3 \mu^{2}+\mu_{5}^{2}\right)+\frac{\mu_{5} T^{2}}{6}\right) \boldsymbol{\omega} .
\end{aligned}
$$

In the above expressions, we introduce the quantities $\mu=$ $\mu_{R}+\mu_{L}, \mu_{5}=\mu_{L}-\mu_{R}, \mathcal{A}_{\mu}^{L}=\mathcal{A}_{\mu}+\mathcal{A}_{\mu}^{5}$, and $\mathcal{A}_{\mu}^{R}=\mathcal{A}_{\mu}-\mathcal{A}_{\mu}^{5}$. However, as discussed in Sec. IV, the covariant electric current does not couple to the electromagnetic field. The proper conserved current that couples to the photon is the consistent current, obtained after including the contribution from the Bardeen-Zumino polynomial and Bardeen counterterm [see Eq. (40)], leading to the expressions

$$
\begin{aligned}
\rho_{e}= & \frac{1}{2 \pi^{2}} \mathbf{b} \cdot \mathbf{B}, \\
\mathbf{j}_{e}= & \frac{\mu}{2 \pi^{2}} \mathbf{B}_{5}+\frac{\mu \mu_{5}}{2 \pi^{2}} \boldsymbol{\omega}+\frac{1}{2 \pi^{2}} \mathbf{E} \times \mathbf{b}, \\
\rho_{5}= & \frac{1}{6 \pi^{2}} \mathbf{b} \cdot \mathbf{B}_{5}, \\
\mathbf{j}_{5}= & \frac{\mu}{2 \pi^{2}} \mathbf{B}+\frac{\mu_{5}}{3 \pi^{2}} \mathbf{B}_{5}+\left(\frac{\mu^{2}+\mu_{5}^{2}}{4 \pi^{2}}+\frac{T^{2}}{12}\right) \boldsymbol{\omega} \\
& +\frac{1}{6 \pi^{2}} \mathbf{E}_{5} \times \mathbf{b} .
\end{aligned}
$$

While arriving at the final expression, we have used $\mathcal{A}_{\mu}^{5}=$ $\left(\mu_{5}, \mathbf{b}\right)$, in order for the model to describe a time-reversal symmetry-breaking Weyl semimetal. In this construction, the separation of two Weyl nodes is $2|\mathbf{b}|$. Note that the covariant current $\mathbf{J}_{e}$ has a contribution $\sim \mu_{5} \mathbf{B}$, which captures the static chiral magnetic effect. However, the Chern-Simons current contains a contribution $-A_{0}^{5} \mathbf{B} \equiv-\mu_{5} \mathbf{B}$, which exactly cancels such a contribution in the conserved current. Therefore, Weyl systems do not exhibit any static chiral magnetic effect in the vector current $\mathbf{j}_{e}$ [70].

After establishing the current operators for the Abelian field theory with $\mathrm{U}(1)_{e} \times \mathrm{U}(1)_{5}$ symmetry, we now construct both the Abelian and non-Abelian currents from the fieldtheoretic description of the multi-Weyl systems, possessing a $\mathrm{U}(1)_{e} \times \mathrm{U}(1)_{5} \times \mathrm{SU}(2)_{e} \times \mathrm{SU}(2)_{5}$ symmetry. At this stage, we introduce the notion of the isospin chemical potentials $\mu_{3}=\mu_{L}^{3}+\mu_{R}^{3}$ and $\mu_{3_{5}}=\mu_{L}^{3}-\mu_{R}^{3}$, respectively, and the corresponding vector and axial gauge fields $\mathbb{A}_{\mu}^{3, L}=\mathbb{A}_{\mu}^{3}+\mathbb{A}_{\mu}^{35}$ and $\mathbb{A}_{\mu}^{3, R}=\mathbb{A}_{\mu}^{3}-\mathbb{A}_{\mu}^{3_{5}}$. The covariant Abelian currents now read

$$
\begin{aligned}
& \mathbf{J}_{e}=\frac{n}{2 \pi^{2}}\left(\mu_{5} \mathbf{B}+\mu \mathbf{B}_{5}\right)+\frac{c(n)}{2 \pi^{2}}\left(\mu_{3_{5}} \mathbf{B}_{3}+\mu_{3} \mathbf{B}_{3_{5}}\right), \\
& \mathbf{J}_{5}=\frac{n}{2 \pi^{2}}\left(\mu \mathbf{B}+\mu_{5} \mathbf{B}_{5}\right)+\frac{c(n)}{2 \pi^{2}}\left(\mu_{3} \mathbf{B}_{3}+\mu_{3_{5}} \mathbf{B}_{3_{5}}\right) .
\end{aligned}
$$

For the sake of simplicity, we ignore here the contribution from the vortical conductivities and the energy current, which we show in Appendix B. To arrive at the consistent currents, we need to evaluate the Chern-Simons polynomial [see Eq. (40)] and add it to the above covariant currents. In particular, for the Abelian charge densities and currents we obtain

$$
\begin{aligned}
\rho_{e}= & \frac{n}{2 \pi^{2}} \mathbf{b} \cdot \mathbf{B} \\
\mathbf{j}_{e}= & \frac{n}{2 \pi^{2}} \mu \mathbf{B}_{5}+\frac{c(n)}{2 \pi^{2}} \mu_{3} \mathbf{B}_{3_{5}}+\frac{n}{2 \pi^{2}} \mathbf{E} \times \mathbf{b} \\
\rho_{5}= & \frac{n}{6 \pi^{2}} \mathbf{b} \cdot \mathbf{B}_{5} \\
\mathbf{j}_{5}= & \frac{n}{2 \pi^{2}} \mu \mathbf{B}+\frac{n}{3 \pi^{2}} \mu_{5} \mathbf{B}_{5}+\frac{c(n)}{2 \pi^{2}} \mu_{3} \mathbf{B}_{3}+\frac{c(n)}{3 \pi^{2}} \mu_{35} \mathbf{B}_{3_{5}} \\
& +\frac{n}{6 \pi^{2}} \mathbf{E}_{5} \times \mathbf{b} .
\end{aligned}
$$

On the other hand, the non-Abelian densities and currents take the forms

$$
\begin{aligned}
\rho_{3}= & \frac{c(n)}{2 \pi^{2}} \mathbf{b} \cdot \mathbf{B}_{3}, \\
\mathbf{j}_{3}= & \frac{c(n)}{2 \pi^{2}} \mu \mathbf{B}_{3_{5}}+\frac{c(n)}{2 \pi^{2}} \mu_{3} \mathbf{B}_{5}+\frac{c(n)}{2 \pi^{2}} \mathbf{E}_{3} \times \mathbf{b}, \\
\rho_{3_{5}}= & \frac{c(n)}{2 \pi^{2}} \mathbf{b} \cdot \mathbf{B}_{3_{5}}+\frac{c(n)}{6 \pi^{2}} \mathbf{b} \cdot \mathbf{B}_{3_{5}}, \\
\mathbf{j}_{3_{5}}= & \frac{c(n)}{2 \pi^{2}} \mu_{3} \mathbf{B}+\frac{c(n)}{3 \pi^{2}} \mu_{3_{5}} \mathbf{B}_{5}+\frac{c(n)}{2 \pi^{2}} \mu \mathbf{B}_{3} \\
& +\frac{c(n)}{3 \pi^{2}} \mu_{5} \mathbf{B}_{3_{5}}+\frac{c(n)}{6 \pi^{2}} \mathbf{E}_{3_{5}} \times \mathbf{b} .
\end{aligned}
$$

In order to relate these currents with the multi-Weyl semimetal we need to take into account the presence of the non-Abelian background field $\mathbb{A}=\Delta\left(0, s_{x}, s_{y}, 0\right)$. The presence of such a background field introduces a non-Abelian magnetic field $\mathbf{B}_{3}=\left(0,0, \Delta^{2}\right)$.

However, there are two issues associated with such a background field that we should address. The first one is associated with the fact that the transport coefficients shown before were computed using the linear-response theory and the actual magnetic field corresponds to a nonlinear contribution; second, the parameter $\Delta$ also breaks the SU(2) symmetry, spoiling the anomaly protection of the current, shown in Eqs. (60)-(63). We deal with this subtlety in Sec. VI. Prior to that, we proceed to anchor some of the predictions from the effective field theory for multi-Weyl semimetals to their lattice realizations.

\section{ANOMALOUS RESPONSES FROM LATTICE MODELS}

In the previous sections, we established the low-energy models (both in the continuum and from the tight-binding models on a cubic lattice) and the effective field theoretic description for multi-Weyl semimetals. We now test the predictions from the effective field theory (see Sec. IV) by computing some specific observables or expectation values of some operators from the lattice regularized models, introduced in Sec. III A. We first focus on the Abelian sector, for which the anomalous Hall effect yields the relations between the charge $\left(\rho_{e}\right)$ and current $\left(\mathbf{j}_{e}\right)$ densities

$$
\rho_{e}=n \frac{e^{2}}{2 \pi^{2}}(\mathbf{b} \cdot \mathbf{B}), \quad \mathbf{j}_{e}=n \frac{e^{2}}{2 \pi^{2}}(\mathbf{b} \times \mathbf{E}),
$$




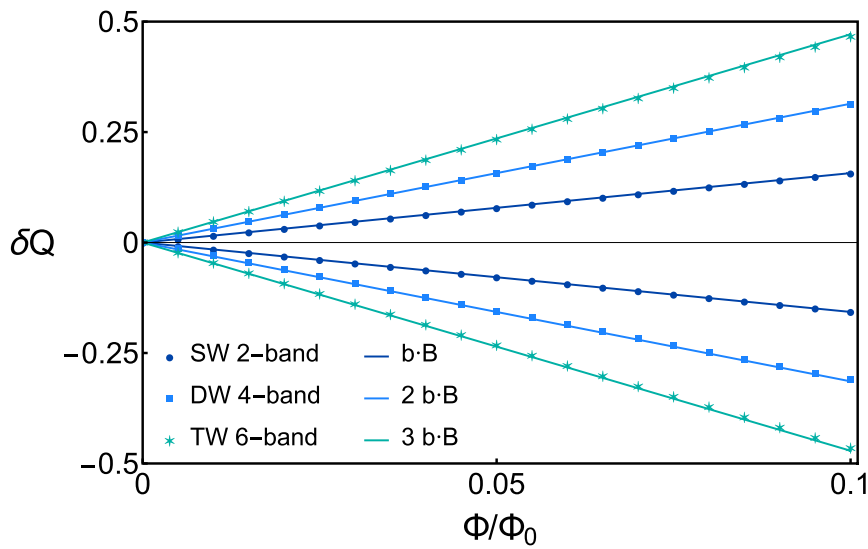

(a)

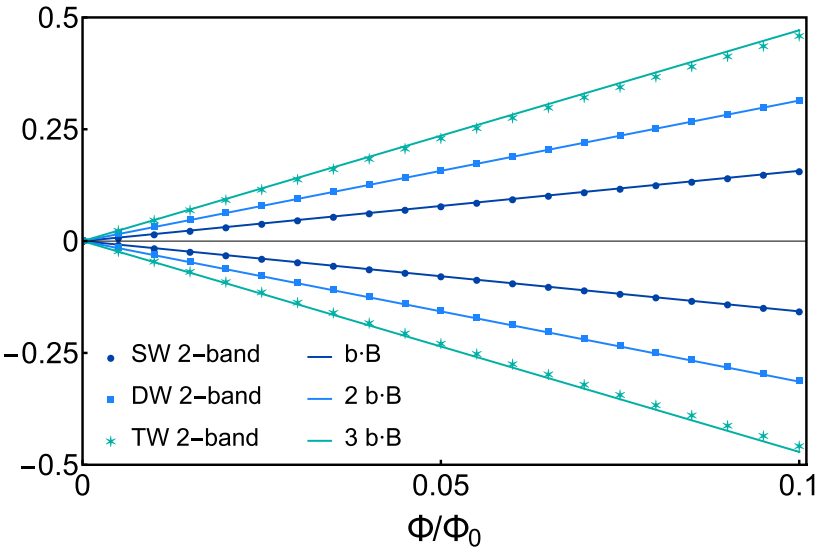

(b)

FIG. 6. Charge density $\delta Q$, measured in units of $e / \pi$, in the vicinity of the flux tubes placed at $x=L / 4$ (yielding $\delta Q>0$ ) and $x=-L / 4$ (yielding $\delta Q<0)$ [see Eq. (65)] for simple Weyl (SW), double Weyl (DW), and triple Weyl (TW) semimetals. Results for simple Weyl semimetal are always obtained from the two-band model. By contrast, for the double- (triple-) Weyl semimetals, we compute $\delta Q$ from (a) four(six-) and (b) two-band models. Here $\Phi$ is the magnetic flux, measured in units of the flux quanta $\Phi_{0}=2 \pi / e$ (in natural units $\hbar=c=1$ ). For numerical analysis, we always set $t=t_{z}=t_{0}=1$ and $L_{z}=200$, where $L_{z}$ is the number of points in the momentum space along the $k_{z}$ direction. Specifically, for (a) we set $\Delta=0.2$ and $L=24$, while for (b) $L=48$, where $L$ is the linear dimension of the system in the $x$ and $y$ directions. The dots correspond to numerically computed values of $\delta Q$, whereas the straight lines follow the relation $\delta Q=n \frac{e}{2 \pi}(\mathbf{b} \cdot \mathbf{B}) \equiv n b\left(\Phi / \Phi_{0}\right)$, where $n$ is the monopole charge of the Weyl nodes, located at $\mathbf{b}=(0,0, \pm b)$, and $b=\pi / 2$. This analysis establishes excellent agreement between the field-theoretic predictions and the scaling of the corresponding observable computed from the lattice models.

where $n$ is the monopole charge, $e$ is the Abelian electric charge, $\mathbf{B}(\mathbf{E})$ is the external magnetic (electric) field, and the Weyl points are located at $\mathbf{b}=(0,0, \pm b)$. In our lattice models, $b=\pi / 2 a$, where $a$ is the lattice spacing, set equal to unity for convenience.

Since the charge and current densities arise, respectively, from the temporal and spatial components of the same ChernSimons current, we only compute $\rho_{e}$ from the lattice model. To this end, we consider a cubic lattice, with $L$ sites in the $x$ and $y$ directions, and preserve translational invariance along the $z$ direction (leaving $k_{z}$ as a good quantum number). We impose periodic boundaries in the $x$ and $y$ directions. The effect of the magnetic field can be incorporated via the Peierls substitution: Hopping terms between lattice sites at $\mathbf{r}_{i}$ and $\mathbf{r}_{f}$ acquire the phase $\exp \left[\frac{2 \pi i}{\Phi_{0}} \int_{\mathbf{r}_{i}}^{\mathbf{r}_{f}} \mathbf{A} \cdot d \mathbf{r}\right]$, where $\mathbf{A}$ is the Abelian vector potential and $\Phi_{0}=h c / e$ is the flux quantum. In what follows, the magnetic field assumes the profile [71]

$$
\mathbf{B}=\Phi\left[\delta\left(x-\frac{L}{4}\right)-\delta\left(x+\frac{L}{4}\right)\right] \delta(y) \hat{z},
$$

where $\Phi$ is the flux produced by the external magnetic field and $\delta$ is the Dirac delta function. For convenience, we work in the Landau gauge, given by

$$
\mathbf{A}=\Phi\left[\Theta\left(x-\frac{L}{4}\right)-\Theta\left(x+\frac{L}{4}\right)\right] \delta(y) \hat{y},
$$

where $\Theta$ is the Heaviside step function.

Upon numerically diagonalizing the tight-binding model in the presence of such a singular magnetic field, we compute the charge accumulation at a given point $(x, y)$ from the expression

$$
\rho_{e}(x, y)=e \sum_{E_{i}<0} \sum_{\alpha} \sum_{k_{z}}\left|\Psi_{\alpha}\left(x, y, k_{z}, E_{i}\right)\right|^{2},
$$

where $\alpha$ is the pseudospin and flavor multi-index, $\Psi_{\alpha}\left(x, y, k_{z}, E_{i}\right)$ is the eigenstate with energy $E_{i}$, and the summation is performed over the ground-state configuration, hence $E_{i}<0$. In order to perform the summation over $k_{z}$, we discretize the interval $k_{z} \in(-\pi, \pi)$ into $L_{z}$ points. The accumulated charge density around the flux tube located at $x=L / 4$ is given by

$$
\delta Q=\sum_{x=1}^{L / 2} \sum_{y=1}^{L} \frac{\rho_{e}(x, y)}{L_{z}}
$$

The scaling of $\delta Q$ with $\Phi$ for multi-Weyl semimetals is shown in Fig. 6.

We now compare the outcomes with the field-theoretic predictions. In natural units $(\hbar=c=1)$, the flux quantum is $\Phi_{0}=2 \pi / e$, and when we measure the accumulated charge density in units of $e / \pi$, the anomaly equation for $\rho_{e}$ or $\delta Q$ from Eq. (64) becomes

$$
\delta Q=n b\left(\frac{\Phi}{\Phi_{0}}\right)
$$

Note that, in numerical analysis, $\rho$ or $\delta Q$ is measured with respect to its expectation value in the absence of the external magnetic field. For simple Weyl semimetals we always compute this quantity from the two-band model. However, for double- (triple-) Weyl semimetals we compute this quantity from the four- (six-) [see Fig. 6(a)] and two-band [see Fig. 6(b)] models. From Fig. 6 we find that the slope of the straight lines in the $\left(\Phi / \Phi_{0}, \delta Q\right)$ plane is given by $n \pi / 2$ for weak enough magnetic fields $\left(B a^{2} \ll 1\right)$, irrespective of the microscopic details. These results establish excellent agreement with the field-theoretic predictions. 


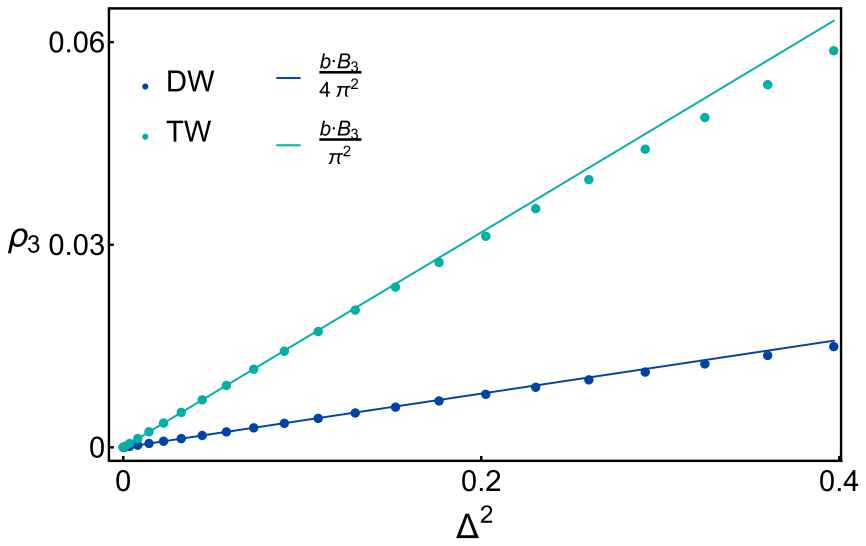

FIG. 7. Non-Abelian or isospin density $\rho_{3}$ as a function of the non-Abelian magnetic field $\Delta^{2}$, computed numerically from the fourand six-band models for the double-Weyl and triple-Weyl semimetals, respectively. For numerical analysis we set $t=t_{z}=t_{0}=1$ and discretize each momentum direction into 41 points in the Brillouin zone. In our construction, $\left|\mathbf{B}_{3}\right|=\Delta^{2}$ and $b=\pi / 2$.

Next we focus on the anomalous Hall effect for the nonAbelian density $\left(\rho_{3}\right)$ and current $\left(\mathbf{J}_{3}\right)$, given by

$$
\rho_{3}=\frac{c(n)}{2 \pi^{2}}\left(\mathbf{b} \cdot \mathbf{B}_{3}\right), \quad \mathbf{j}_{3}=\frac{c(n)}{2 \pi^{2}}\left(\mathbf{E}_{3} \times \mathbf{b}\right),
$$

where $\mathbf{B}_{3}\left(\mathbf{E}_{3}\right)$ is the non-Abelian magnetic (electric) field, pointing in the $z$ direction in the flavor/isospin space, and $c(n)=\frac{1}{2}$ (2) for $n=2$ (3). Recall that the above set of nonAbelian anomaly equations are only germane for multi-Weyl semimetals with $n>1$. To test the validity of the fieldtheoretic predictions, we compute the non-Abelian or isospin density $\rho_{3}$ from the lattice models for double- and triple-Weyl fermions. Note that the presence of the non-Abelian field is transparent only in the four- (six-) band models for double (triple) Weyl semimetals, and in particular $\mathbf{B}_{3}=\left(0,0, \Delta^{2}\right)$. Thus, we compute $\rho_{3}$ from these models only. Since $\mathbf{B}_{3}$ is an intrinsic homogenous field, the computation is performed in the momentum space representation of the corresponding lattice models. In particular, $\rho_{3}$ is computed from the expression

$$
\rho_{3}=\frac{1}{L_{x} L_{y} L_{z}} \sum_{E_{i}<0} \sum_{\mathbf{k}}\left\langle\Psi\left(E_{i}, \mathbf{k}\right)\left|\tau_{0} \otimes s_{3}^{n}\right| \Psi\left(E_{i}, \mathbf{k}\right)\right\rangle,
$$

where $L_{j}$ is the number of sites in the $k_{j}$ direction in the momentum space, where $j=x, y, z$. The results are displayed in Fig. 7.

Numerically evaluated $\rho_{3}$ is compared with $\Delta^{2}$ (strength of the non-Abelian magnetic field $\mathbf{B}_{3}$ ), and we find an excellent linear dependence of $\rho_{3}$ on $\Delta^{2}$ for $n=2$ and 3 for small $\Delta^{2}$ such that $\Delta a \ll 1$. The slopes of the linear fits are given, respectively, by $b / 4 \pi^{2} \equiv 1 / 8 \pi^{3}$ and $b / \pi^{2} \equiv 1 / 2 \pi^{3}$ for $n=2$ and 3 , since in our lattice construction $b=\pi / 2$. This observation establishes excellent agreement between the field-theoretic predictions on the non-Abelian anomaly for multi-Weyl semimetals and the numerical findings from the lattice models.

In the context of non-Abelian anomaly, a comment is due at this stage. We note that the isospin density operator $\tau_{0} \otimes s_{3}^{n}$ defined in the four- (six-) band models for double(triple-) Weyl semimetal reduces to $\sigma_{3}$ in their low-energy sector (emergent two-band description). In principle, we can compute the expectation value for this operator $\sigma_{3}$ from the two-band models for the double- and triple-Weyl semimetals. However, note that the operator $\sigma_{3}$ can be obtained by projecting multiple operators defined in the four- or sixband model. Hence, the inverse of the projection operation is not unique. Consequently, even though the expectation value of $\sigma_{3}$, namely, $\left\langle\sigma_{3}\right\rangle$, is finite, we find that in general it is much larger than the pure non-Abelian anomaly contribution. Presently, there is no known procedure to isolate the contribution in $\left\langle\sigma_{3}\right\rangle$ arising purely from the non-Abelian anomaly. This is the reason we do not display the results on the scaling of $\left\langle\sigma_{3}\right\rangle$ with $\left|\mathbf{B}_{3}\right|=\Delta^{2}$.

\section{ANOMALOUS TRANSPORT FROM HOLOGRAPHY}

As discussed in Sec. IV, the presence of the background non-Abelian gauge field breaks the original global symmetry group. For the sake of simplicity, we consider only lefthanded matter fields. In this case, the starting symmetry group is $\mathrm{SO}(3,1) \times \mathrm{SU}(2)_{L} \times \mathrm{U}(1)_{L}$, which gets broken down to $\mathrm{SO}(1,1) \times \mathrm{U}(1)_{3_{L}} \times \mathrm{U}(1)_{L}$ by the background gauge field. This explicit symmetry breaking generates a renormalizationgroup (RG) flow from a conformal field theory in the ultraviolet (UV) to an anisotropic system in the infrared (IR), as the one described by the effective multi-Weyl Hamiltonian in Eq. (2). Therefore, along the RG trajectory the anomalous conductivities are not necessarily anomaly protected. However, in some cases the IR conductivities show a universal behavior $[38,39]$.

In order to understand the one-point functions of the currents at very low energies, it is necessary to select a specific model. We focus on a strongly coupled theory with a holographic dual, considering the simplicity of the computations of one-point functions in this case. A second reason for selecting a holographic model is associated with the ambiguities we may encounter related to different regularization schemes $[11,13]$ in perturbative quantum field theories. Fortunately, in holography these ambiguities are not present due to the existence of a natural regulator [location of the anti-de Sitter (AdS) boundary]. As a matter of fact, holography has been a vital tool for the understanding of the anomaly-induced transport $[12,42,72,73]$, and our main goal is to qualitatively demonstrate whether the predicted transport coefficients survive along the RG flow.

As our first approach to the problem, and for simplicity, we consider only the response of the charged current to external gauge fields. We leave the study of chiral vortical conductivities and the response in the energy momentum tensor for future investigation. That allows us to make the probe approximation, in which the bulk gauge fields do not backreact on the geometry. As a consequence, the mixed gauge-gravitational anomaly decouples. However, as already seen in previous sections, the mixed anomaly is relevant for the chiral vortical or chiral magnetic effects in the energy current.

In holography, the problem of quantum anomalies is well understood and their presence is realized via the introduction 
of Chern-Simons terms in the bulk action. Our perspective is completely phenomenological, i.e., bottom up. We assume the existence of a large $N_{c}$ (color number) and strongly coupled gauge theory with a holographic dual and with the same anomaly structure as in our multi-Weyl system. Therefore, the simplest holographic model we can construct has the form

$$
\begin{aligned}
S= & -\int \operatorname{Tr}\left[\frac{1}{2 n} \mathcal{F} \wedge^{\star} \mathcal{F}+\frac{1}{2 c(n)} G \wedge^{\star} G\right. \\
& \left.+\lambda\left(A \wedge(d A)^{2}+\frac{3}{2} A^{3} \wedge d A+\frac{3}{5} A^{5}\right)\right],
\end{aligned}
$$

where the gauge fields are defined as

$$
\mathcal{A}=\mathcal{A}^{0} s_{0}, \quad \mathbb{A}=\mathbb{A}^{i} s_{i}, \quad A=\mathcal{A}+\mathbb{A},
$$

with $s_{a}=\left(s_{0}, s_{i}\right)$ the identity and SU(2) generators introduced in Sec. II. The corresponding field strengths associated with the gauge fields are

$$
\mathcal{F}=d \mathcal{A}, \quad G=d \mathbb{A}-i \mathbb{A}^{2}, \quad F=\mathcal{F}+G .
$$

We seek to compute the anomalous currents at finite temperature. To do so, we need a finite-temperature background geometry, which we choose to be the Schwarzschild-AdS black hole

$$
d s^{2}=\frac{1}{r^{2}}\left(-u(r) d t^{2}+\frac{1}{u(r)} d r^{2}+d x^{2}+d y^{2}+d z^{2}\right),
$$

with the horizon at $r_{h}=1$ and the blackening factor $u(r)=$ $1-r^{2}$. In these units the Hawking temperature is given by $T=\pi^{-1}$.

To connect the Chern-Simons term in the action (72) with the anomaly, we use the fact that the gauge-gravity duality establishes that the on-shell action $S$ corresponds to the boundary quantum field theory effective action $W[A]$, with

$$
W[A]=S_{\text {on-shell }}[A] .
$$

Therefore, after performing a bulk gauge transformation $\delta_{\theta} A_{M}=-\mathcal{D}_{M} \theta$, where $M=0,1, \ldots, 4$ and $x^{M}=$ $(t, x, y, z, r)$, we reproduce the expression

$$
\delta_{\theta} W(A)=\int d^{4} x \theta^{a} G^{a}[A],
$$

where $G^{a}$ is the anomaly [see the right-hand side of Eq. (28)]. This computation shows how the anomaly is realized within the holographic setup and allows us to set the coupling $\lambda$,

$$
\lambda=\frac{N_{c}}{24 \pi^{2}} .
$$

As the next step we define the holographic one-point functions by taking functional derivatives of Eq. (76). In particular, derivatives with respect to the gauge fields generate the (unrenormalized) consistent charged currents

$$
\tilde{J}_{a}^{\mu}=\frac{\delta S}{\delta A_{\mu}^{a}}=\left.\sqrt{-g}\left(2 F_{a}^{r \mu}-K_{a}^{\mu}[A]\right)\right|_{\text {boundary }},
$$

where

$$
K_{a}^{\mu}[A]=-\frac{\lambda}{2} \epsilon^{\mu \nu \rho \sigma} \operatorname{Tr}\left[s_{a}\left(\left\{A_{\nu}, F_{\rho \sigma}\right\}-A_{\nu} A_{\rho} A_{\sigma}\right)\right]
$$

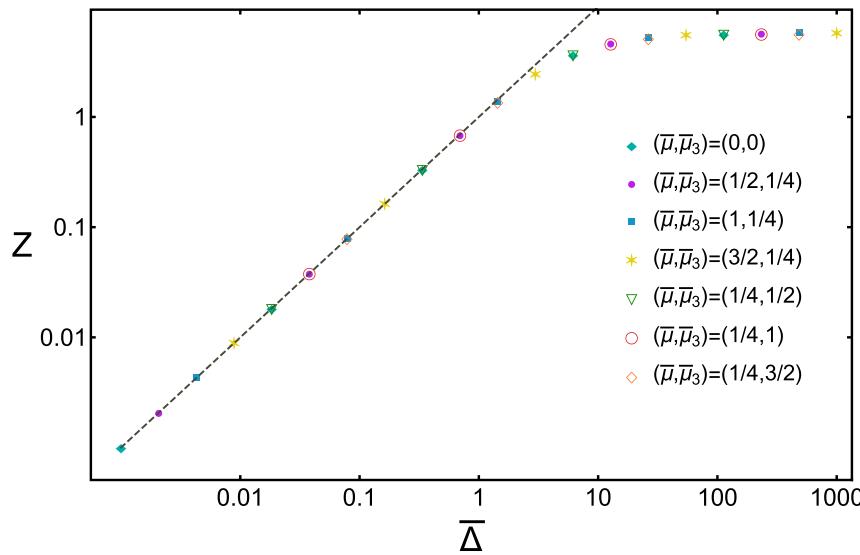

FIG. 8. Renormalization of the background non-Abelian gauge field for different chemical potentials (see the inset) as a function of $\bar{\Delta}$. The IR values correspond to $\bar{\Delta} \rightarrow \infty$. The dashed line corresponds to a linear fit $Z=\bar{\Delta}$.

is precisely the Bardeen-Zumino polynomial. Consequently, we can read from Eq. (79) the holographic definition of the (unrenormalized) covariant current

$$
J_{a}^{\mu}=\left.2 \sqrt{-g} F_{a}^{r \mu}\right|_{\text {boundary }} .
$$

After defining the model, we introduce the bulk gauge field ansatz, which is dual to the boundary field theory at finite density and temperature. Furthermore, in order to study the anomaly-induced currents, we introduce an external background magnetic field $\mathbf{B}=(0,0, B)$ and the symmetrybreaking non-Abelian gauge field responsible for the multiWeyl spectrum in the weakly coupled model. Thus, the bulk gauge field at the boundary has to take the value

$$
A\left(r_{b}\right)=\left(\mu s_{0}+\mu_{3} s_{z}\right) d t+\Delta\left(s_{x} d x+s_{y} d y\right)+x B s_{0} d y .
$$

With all these ingredients, the simplest ansatz we need to consider takes the form

$$
\begin{aligned}
A(r)= & {\left[A_{t}(r) s_{0}+A_{t}^{3}(r) s_{z}\right] d t+\mathcal{Q}(r)\left(s_{x} d x+s_{y} d y\right) } \\
& +\left[A_{z}(r) s_{0}+A_{z}^{3}(r) s_{z}\right] d z+x B s_{0} d y .
\end{aligned}
$$

This ansatz needs to be plugged into the equations of motion and solved by imposing the boundary conditions (82) at the boundary and regularity in the interior of the space-time except for the fields $A_{t}$ and $A_{t}^{3}$, which has to vanish. In Appendix $\mathrm{C}$ we show the explicit form of the equations of motion.

The first conclusion we make after writing down the equations of motion is that the Abelian covariant current can be obtained analytically and takes its universal form

$$
J^{z}=\frac{n}{4 \pi^{2}} \mu B+\frac{c(n)}{4 \pi^{2}} \mu_{3} \Delta^{2} .
$$

On the other hand, the non-Abelian sector needs to be solved numerically.

Before showing the results for the non-Abelian current we discuss some aspects of the boundary sources $\mu, \mu_{3}$, and $\Delta$. They introduce a deformation of the UV conformal field theory as

$$
\mathcal{L}=\mathcal{L}_{\mathrm{CFT}}+\mu Q+\mu_{3} Q^{3}+\Delta\left(\delta_{x}^{\mu} j_{\mu}^{1}+\delta_{y}^{\mu} j_{\mu}^{2}\right) .
$$




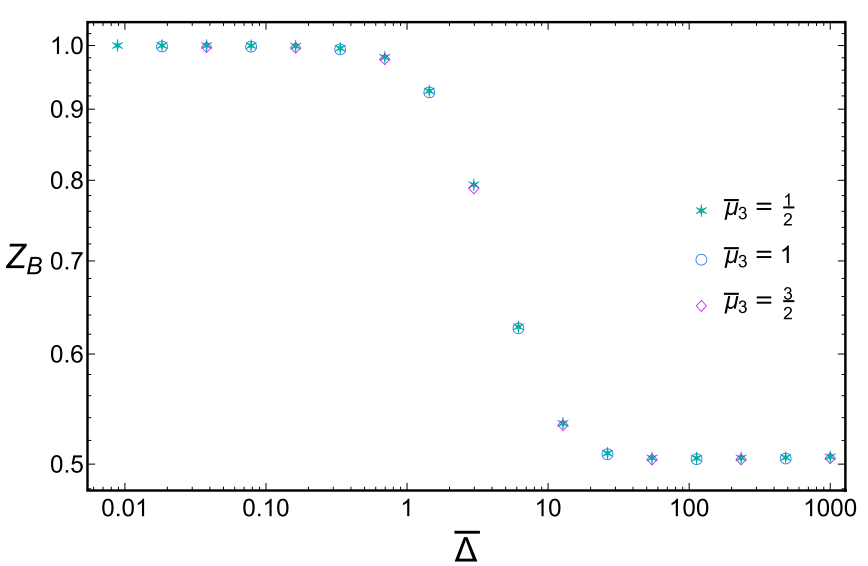

FIG. 9. Isospin chiral magnetic conductivity as a function of $\bar{\Delta}$ for vanishing chemical potential $\mu$.

In particular, the charge $Q$ remains conserved (modulo the anomaly). However, by the presence of $\Delta$, all the charges $Q^{i}$ are not conserved, because the $\mathrm{SU}(2)$ group is explicitly broken. Hence, $\Delta$ has to be understood as a coupling constant, which gets renormalized along the RG flow, since no symmetry protects its value. For that reason, we define the IR renormalized $\Delta$ as

$$
\Delta_{\mathrm{IR}}=\mathcal{Q}\left(r_{h}\right)
$$

At this point it is convenient to introduce the set of adimensional variables

$$
\bar{\Delta}=\frac{\Delta}{T}, \quad \bar{\mu}=\frac{\mu}{T}, \quad \bar{\mu}_{3}=\frac{\mu_{3}}{T} .
$$

In Fig. 8 we show the renormalized $\Delta_{\text {IR }}$ for several values of the chemical potentials and find it to be independent of $\bar{\mu}$ and $\bar{\mu}_{3}$. Therefore, we infer that $\Delta_{\mathrm{IR}}=T Z(\bar{\Delta})$, with

$$
Z(\bar{\Delta}) \approx \begin{cases}\bar{\Delta} & \text { for } \bar{\Delta} \ll 1 \\ 5.7584 & \text { for } \bar{\Delta} \gg 1 .\end{cases}
$$

From the above dependences, we conclude that $\Delta_{\mathrm{IR}} \sim 5.8 T$ for small enough temperatures.

We now compute the isospin current $\left(J_{3}^{z}\right)$ in the presence of either the isospin chemical potential $\left(\mu_{3}\right)$ or chemical potential $(\mu)$. In the former case, we find the generic behavior

$$
J_{3}^{z}=Z_{B}(\bar{\Delta}) \frac{c(n) \mu_{3}}{4 \pi^{2}} B,
$$

and the function $Z_{B}$ appears to be independent of $\mu_{3}$, as can be seen in Fig. 9. Numerical analysis suggests that $Z_{B}(\bar{\Delta})$ has the functional form

$$
Z_{B}(\bar{\Delta}) \approx \begin{cases}1 & \text { for } \bar{\Delta} \ll 1 \\ 0.506 & \text { for } \bar{\Delta} \gg 1,\end{cases}
$$

which implies that at low temperatures the isospin chiral magnetic effect is reduced by a factor of 2 (approximately), yielding

$$
J_{3}^{z} \approx 0.5 \frac{n}{4 \pi^{2}} \mu_{3} B .
$$

Next we study the system only in the presence of the Abelian chemical potential $\mu$. In this case, the isospin current takes the

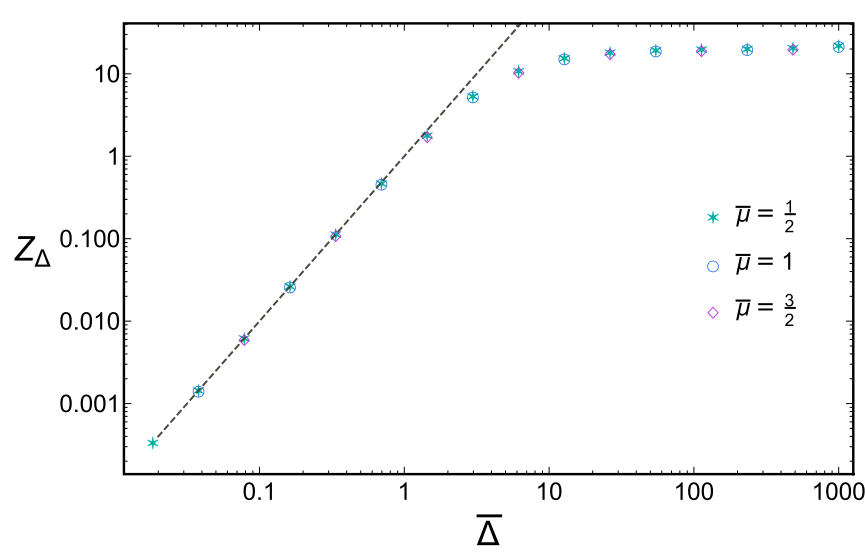

FIG. 10. Isospin current as a function of $\bar{\Delta}$ for vanishing isospin chemical potential $\mu_{3}$. The dashed line corresponds to a quadratic fitting $Z_{\Delta}=\bar{\Delta}^{2}$.

form

$$
J_{3}^{z}=Z_{\Delta}(\bar{\Delta}) \frac{c(n)}{4 \pi^{2}} \mu T^{2} .
$$

The functional dependence of $Z_{\Delta}$ on $\bar{\Delta}$ is shown in Fig. 10 and it depends only on the symmetry-breaking parameter $\bar{\Delta}$. In the two asymptotic regimes, it is characterized as

$$
Z_{\Delta}(\bar{\Delta}) \approx \begin{cases}\bar{\Delta}^{2} & \text { for } \bar{\Delta} \ll 1 \\ 21.762 & \text { for } \bar{\Delta} \gg 1\end{cases}
$$

As expected, at high enough temperatures the current takes the universal value set by the anomaly

$$
J_{3}^{z} \approx \frac{c(n)}{4 \pi^{2}} \mu \Delta^{2} .
$$

However, at very low temperatures it has a different behavior, given by

$$
J_{3}^{z} \approx 21.8 \frac{c(n)}{4 \pi^{2}} \mu T^{2},
$$

which is set by the temperature instead of the parameter $\Delta$. Since the renormalized IR value of the non-Abelian gauge field is $\Delta_{\mathrm{IR}} \approx 5.8 T$ [see Eq. (88)], the current can alternatively be written as

$$
J_{3}^{z} \approx 0.6 \frac{c(n)}{4 \pi^{2}} \mu \Delta_{\mathrm{IR}}^{2} .
$$

At this point a comment is due regarding the probe approximation. Ignoring the backreaction of the gauge fields on the space-time geometry is a valid approximation, as long as the temperature is not "too low." Therefore, some numerical deviations of the coefficients [see Eqs. (91) and (96)] can be expected for the IR conductivities.

Therefore, at this point we cannot conclude regarding the universalities associated with the conductivities. Nonetheless, the important fact is that their coefficients are of order one, which strongly suggests that even in the IR low-energy model the anomalous transport survives in the isospin current, which is directly connected to the $\mathrm{SO}(2)$ rotational invariance of the low-energy sector. 


\section{DISCUSSION AND FUTURE DIRECTIONS}

The primary goal of the present work was to develop a comprehensive understanding of anomalies in multi-Weyl semimetals. The lack of Lorentz symmetry (stemming from the nonlinear band dispersion) yields a complex and rich structure in the theory, which motivated us to find a suitable generalization of these models in order to shed light on the desired physical phenomena. The appropriate formulation was presented in Sec. II, revealing the presence of an underlying non-Abelian symmetry and associated anomalies. This construction is based on $n$ copies of simple Weyl fermions, coupled via a particular spin-orbit coupling that preserves certain discrete crystalline symmetry, but breaks the Lorentz invariance and couples with linearly dispersing chiral fermions as a static SU(2) gauge field. As a result, the multi-Weyl spectra are recovered at low energies. Our study aimed at a systematic analysis of mixed Abelian and non-Abelian anomalies and associated transport, which we achieved by employing various approaches available in condensed-matter and high-energy physics.

We introduced both two-band models for general Weyl semimetals (with $n=1,2,3$ ) and the four- (six-) band model for the double- (triple-) Weyl semimetals on a cubic lattice in Sec. III A. These models produced the correct low-energy descriptions for multi-Weyl semimetals (compare Figs. 2 and 3 ), as well as the bulk-boundary correspondence, encoded in the number $(n)$ of topologically protected Fermi arc surface states (see Sec. III B and Figs. 4 and 5). At this point we recessed the lattice simulations to construct an effective field theory for multi-Weyl systems.

The key observation while arriving at the effective field theory is the presence of a non-Abelian U(2) flavor symmetry, which is controlled by a background field and responsible for the multi-Weyl dispersion at low energies. This representation allowed us to write a Lagrangian in the form of free fermions coupled to a non-Abelian background field. Subsequently, we employed powerful techniques of quantum field theory to identify Abelian and non-Abelian currents that exhibit anomalous nonconservation due to pertinent gauge and gravitational anomalies. Such an elegant formulation allowed us to relate the anomaly structure in multi-Weyl semimetals to the ones previously known for Lorentz symmetric systems and at the same time to bypass cumbersome computational steps. Most importantly, we managed to extract possible transport contributions that stem from anomalies (such as non-Abelian generalization of anomalous Hall conductivity) and are unique to multi-Weyl systems.

We confirmed some of the field theoretic predictions from the lattice models and the gauge-gravity duality. In particular, we numerically computed the regular and isospin charge accumulations as a function of the Abelian and non-Abelian magnetic fields, respectively, for both double- and triple-Weyl semimetals. Remarkably, the numerical outcomes displayed excellent agreement with the field theory, at least when the field strengths were sufficiently weak (see Figs. 6 and 7). Finally, we employed the holographic techniques to study a strongly coupled version of multi-Weyl semimetals. From holography, we analyzed the corresponding renormalizationgroup flow in a particular theory that is dual to the Maxwell-Einstein system with a non-Abelian Chern-Simons term and showed that the non-Abelian anomalous transport coefficients, even though they get renormalized, remain finite in the infrared regime.

There are many interesting possible future outgrowths of the present investigation. We now highlight some of the most exciting ones. Our theoretical analysis suggests possible experimental ramifications of non-Abelian anomaly in magnetotransport. In order to capture its signatures on magnetoresistance, one needs to systematically develop a semiclassical framework by taking into account the non-Abelian currents (see, for example, Refs. [74,75]).

Yet another avenue to explore is to demonstrate or verify field-theoretic predictions from concrete lattice models. In this work we introduced only the simple setup with a background non-Abelian field and computed the regular and isospin charge accumulation in the system. For example, in the same setup one can also introduce chiral chemical potential by adding a term $\mu_{5} \sin \left(k_{z} a\right)$ in the lattice model. Furthermore, one can perform detailed numerical investigations in the presence of axial magnetic fields (obtained via local deformations of hopping parameters) or gravitational fields in multi-Weyl semimetals.

We also note that the transport coefficients associated with the non-Abelian current get renormalized and thus are generically different in the infrared and ultraviolet regimes. Therefore, a detailed field-theoretic analysis leading to the renormalization-group flow of these coefficients using the Feynman diagrammatic expansion is due, which we leave for future investigation.

Our field-theoretic analysis can be extended for multifold fermions [44,45], for which the irreducible band representation transforms under a spin- $S$ representation, where $S$ can be a half-integer or an integer. At the Hamiltonian level this can be accomplished by replacing two-dimensional Pauli matrices $(\boldsymbol{\tau})$ by $(2 S+1)$-dimensional spin- $S$ matrices [61]. A systematic derivation of the effective field theory for multifold fermions is left for future investigation. Finally, we can extend the holographic studies of a multi-Weyl systems to compute the vortical conductivities and energy current, for which the backreaction from the gauge field on the metric should accounted for.

\section{ACKNOWLEDGMENTS}

F.P.-B. and P.S. acknowledge useful conversations with Karl Landsteiner and Maria Vozmediano and the hospitality of Nordita. P.S. was supported by the Deutsche Forschungsgemeinschaft via the Leibniz Programm. F.P.-B., P.S. and R.M.A.D. acknowledge DFG through Würzburg-Dresden Cluster of Excellence on Complexity and Topology in Quantum Matter - ct.qmat (EXC 2147, project-id 39085490). F.P.B. acknowledges the Quantum Matter Academy of ct.qmat for support. B.R. was partially supported by the Start-up Grant from Lehigh University.

\section{APPENDIX A: LOW-ENERGY HAMILTONIAN FOR MULTI-WEYL FERMIONS}

We devote this Appendix to deriving the low-energy Hamiltonian for double- and triple-Weyl semimetals [see 
Eq. (2)] starting from two and three coupled copies of simple Weyl fermions [see Eq. (8)], respectively. Accordingly, we introduce four- and six-component spinors

$$
\begin{aligned}
& \Psi^{\top}(\mathbf{p})_{\mathrm{DW}}=\left[c_{1, \uparrow}, c_{1, \downarrow}, c_{2, \uparrow}, c_{2, \downarrow}\right](\mathbf{p}), \\
& \Psi^{\top}(\mathbf{p})_{\mathrm{TW}}=\left[c_{1, \uparrow}, c_{1, \downarrow}, c_{2, \uparrow}, c_{2, \downarrow}, c_{3, \uparrow}, c_{3, \downarrow}\right](\mathbf{p})
\end{aligned}
$$

for these two systems, where $c_{j, \tau}(\mathbf{p})$ is the fermionic annihilation operator with pseudospin projection $\tau=\uparrow, \downarrow$, flavor index $j=1,2,3$, and momentum $\mathbf{p}$, measured from the Weyl node, located at $\mathbf{p}=0$. In this basis, the Hamiltonian operators yielding double- and triple-Weyl fermions at low energies take, respectively, the forms

$$
\begin{aligned}
& H_{\mathrm{DW}}=\left[\begin{array}{c|c}
v_{\perp}\left(\boldsymbol{\tau}_{\perp} \cdot \mathbf{p}_{\perp}\right)+v \tau_{z} p_{z} & \Delta\left(\tau_{1}-i \tau_{2}\right) / 2 \\
\hline \Delta\left(\tau_{1}+i \tau_{2}\right) / 2 & v_{\perp}\left(\boldsymbol{\tau}_{\perp} \cdot \mathbf{p}_{\perp}\right)+v \tau_{z} p_{z}
\end{array}\right], \\
& H_{\mathrm{TW}}=\left[\begin{array}{c|c|c}
v_{\perp}\left(\boldsymbol{\tau}_{\perp} \cdot \mathbf{p}_{\perp}\right)+v \tau_{z} p_{z} & \Delta\left(\tau_{1}-i \tau_{2}\right) / 2 & \hat{0}_{2 \times 2} \\
\hline \Delta\left(\tau_{1}+i \tau_{2}\right) / 2 & v_{\perp}\left(\boldsymbol{\tau}_{\perp} \cdot \mathbf{p}_{\perp}\right)+v \tau_{z} p_{z} & \Delta\left(\tau_{1}-i \tau_{2}\right) / 2 \\
\hline \hat{0}_{2 \times 2} & \Delta\left(\tau_{1}+i \tau_{2}\right) / 2 & v_{\perp}\left(\boldsymbol{\tau}_{\perp} \cdot \mathbf{p}_{\perp}\right)+v \tau_{z} p_{z}
\end{array}\right],
\end{aligned}
$$

where $\mathbf{p}_{\perp}=\left(p_{x}, p_{y}\right), \boldsymbol{\tau}_{\perp}=\left(\tau_{1}, \tau_{2}\right)$, and $\hat{0}_{2 \times 2}$ represents a two-dimensional null matrix. Note that for $\mathbf{p}=0, c_{1, \downarrow}$ and $c_{2, \uparrow}$ degrees of freedom are gapped and placed at energies $\pm \Delta$ for the double-Weyl system. For the triple-Weyl system $c_{1, \downarrow}$ and $c_{3, \uparrow}\left(c_{2, \uparrow}\right.$ and $c_{3, \downarrow}$ ) are placed at energy $+\Delta(-\Delta)$. Hence, these degrees of freedom do not participate (approximately) in the lowenergy dynamics of the multi-Weyl systems. We therefore integrate them out in order to arrive at the effective low-energy models. If we denote the spinor bases for low- and high-energy degrees of freedom for the double- (triple-) Weyl system by $\Psi_{\mathrm{DW}, \mathrm{L}}(\mathbf{p})\left[\Psi_{\mathrm{TW}, \mathrm{L}}(\mathbf{p})\right]$ and $\Psi_{\mathrm{DW}, \mathrm{H}}(\mathbf{p})\left[\Psi_{\mathrm{TW}, \mathrm{H}}(\mathbf{p})\right]$, respectively, then

$$
\begin{aligned}
& \Psi_{\mathrm{DW}, \mathrm{L}}^{\top}(\mathbf{p})=\left[c_{1, \uparrow}, c_{2, \downarrow}\right](\mathbf{p}), \quad \Psi_{\mathrm{TW}, \mathrm{L}}^{\top}(\mathbf{p})=\left[c_{1, \uparrow}, c_{3, \downarrow}\right](\mathbf{p}), \\
& \Psi_{\mathrm{DW}, \mathrm{H}}^{\top}(\mathbf{p})=\left[c_{1, \downarrow}, c_{2, \uparrow}\right](\mathbf{p}), \\
& \Psi_{\mathrm{TW}, \mathrm{H}}^{\top}(\mathbf{p})=\left[c_{1, \downarrow}, c_{2, \uparrow}, c_{2, \downarrow}, c_{3, \uparrow}\right](\mathbf{p}) .
\end{aligned}
$$

One can integrate out the split-off bands in the path-integral formalism in the following way. The imaginary-time partition function for the multi-Weyl system reads

$$
\begin{aligned}
Z_{a}= & \int \mathcal{D} \Psi_{a, L}^{\dagger} \mathcal{D} \Psi_{a, L} \exp \left[-\int d \tau \Psi_{a, L}^{\dagger}\left(\partial_{\tau}+H_{L L}^{a}\right) \Psi_{a, L}\right] \int \mathcal{D} \Psi_{a, H}^{\dagger} \mathcal{D} \Psi_{a, H} \\
& \times \exp \left[-\int d \tau\left\{\Psi_{a, H}^{\dagger}\left(\partial_{\tau}+H_{H H}^{a}\right) \Psi_{a, H}+\Psi_{a, L}^{\dagger} H_{L H}^{a} \Psi_{a, H}+\Psi_{a, H}^{\dagger} H_{H L}^{a} \Psi_{a, L}\right\}\right],
\end{aligned}
$$

where $a=\mathrm{DW}, \mathrm{TW}$, and

$$
\begin{aligned}
H_{L L}^{\mathrm{DW}} & =\left[\begin{array}{cc}
v p_{z} & 0 \\
0 & -v p_{z}
\end{array}\right]=H_{L L}^{\mathrm{TW}}, \quad H_{H H}^{\mathrm{DW}}=\left[\begin{array}{cc}
-v p_{z} & \Delta \\
\Delta & v p_{z}
\end{array}\right], \quad H_{L H}^{\mathrm{DW}}=\left[\begin{array}{cc}
f\left(\mathbf{p}_{\perp}\right) & 0 \\
0 & f^{*}\left(\mathbf{p}_{\perp}\right)
\end{array}\right], \quad H_{H L}^{\mathrm{DW}}=\left(H_{L H}^{\mathrm{DW}}\right)^{\dagger}, \\
H_{H H}^{\mathrm{TW}} & =\left[\begin{array}{cccc}
-v p_{z} & \Delta & 0 & 0 \\
\Delta & v p_{z} & f\left(\mathbf{p}_{\perp}\right) & 0 \\
0 & f^{*}\left(\mathbf{p}_{\perp}\right) & -v p_{z} & 0 \\
0 & 0 & \Delta & v p_{z}
\end{array}\right], \quad H_{L H}^{\mathrm{TW}}=\left[\begin{array}{cccc}
f\left(\mathbf{p}_{\perp}\right) & 0 & 0 & 0 \\
0 & 0 & 0 & f^{*}\left(\mathbf{p}_{\perp}\right)
\end{array}\right] .
\end{aligned}
$$

In the above expressions $f\left(\mathbf{p}_{\perp}\right)=v\left(p_{x}-i p_{y}\right)$. Upon integrating out the high-energy degrees of freedom, we arrive at the renormalized partition function for the low-energy modes

$$
Z_{a}^{L}=\int \mathcal{D} \Psi_{a, L}^{\dagger} \mathcal{D} \Psi_{a, L} \exp \left[-\int d \tau \Psi_{a, L}^{\dagger}\left(\partial_{\tau}+H_{L L}^{a}-H^{a} G_{H H}^{a}\left(i \omega_{n}\right) H_{H L}^{a}\right) \Psi_{a, L}\right],
$$

where $G_{H H}^{a}\left(i \omega_{n}\right)=\left(i \omega_{n}-H_{H H}^{a}\right)^{-1}$ and $\omega_{n}$ is the Matsubara frequency. Setting $\omega_{n}=0$, from the above expression we arrive at the renormalized Hamiltonian $\left(H_{L L}^{a, \text { Ren }}\right)$ in terms of the low-energy modes

$$
H_{L L}^{a, \text { ren }}=H_{L L}^{a}-H^{a} G_{H H}^{a}(0) H_{H L}^{a} .
$$

After some lengthy but straightforward algebra we find

$$
H_{L L}^{\mathrm{DW}, \mathrm{ren}}=v p_{z} \tau_{3}-\frac{v_{\perp}^{2} \Delta}{\Delta^{2}+v^{2} p_{z}^{2}}\left[\left(p_{x}^{2}-p_{y}^{2}\right) \tau_{1}+2 p_{x} p_{y} \tau_{2}\right]+v p_{z}\left[\frac{v_{\perp}^{2} k_{\perp}^{2}}{\Delta^{2}+v^{2} p_{z}^{2}}\right] \tau_{0},
$$




$$
\begin{aligned}
H_{L L}^{\mathrm{TW}, \text { ren }}= & v p_{z} \tau_{3}+\frac{v_{\perp}^{3} \Delta^{3}}{\left[\left(\Delta^{2}+v^{2} p_{z}^{2}\right)^{2}-v^{2} v_{\perp}^{2} k_{z}^{2} k_{\perp}^{2}\right]}\left[p_{x}\left(p_{x}^{2}-3 p_{y}^{2}\right) \tau_{1}+p_{y}\left(p_{y}^{2}-3 p_{x}^{2}\right) \tau_{2}\right] \\
& +v p_{z}\left[v_{\perp}^{2} p_{\perp}^{2} \frac{v_{\perp}^{2} p_{\perp}^{2}-v_{z}^{2} p_{z}^{2}-\Delta^{2}}{\left(\Delta^{2}+v^{2} p_{z}^{2}\right)^{2}-v^{2} v_{\perp}^{2} k_{z}^{2} k_{\perp}^{2}}\right] \tau_{0} .
\end{aligned}
$$

Note that the particle-hole asymmetric terms (proportional to $\tau_{0}$ ) vanish at the Weyl nodes, located at $\mathbf{p}=0$. Then for $\Delta \gg$ $v_{\perp}\left|\mathbf{p}_{\perp}\right|, v p_{z}$ we arrive at the low-energy Hamiltonian for double- and triple-Weyl semimetal with $\alpha_{n}=v_{\perp}^{n} / \Delta^{n-1}$ for $n=2$ and 3 , respectively (see Sec. II).

\section{APPENDIX B: CHIRAL VORTICAL CONDUCTIVITIES}

In this Appendix we display the transport coefficients omitted in Sec. IV. In particular, the chiral vortical effect in the Abelian currents is given by

$$
\begin{aligned}
& \mathbf{J}_{e}=\left[\frac{n}{2 \pi^{2}} \mu \mu_{5}+c(n) \mu_{3} \mu_{3_{5}}\right] \boldsymbol{\omega}, \\
& \mathbf{J}_{5}=\left[\frac{n}{4 \pi^{2}}\left(\mu^{2}+\mu_{5}^{2}+\frac{\pi^{2} T^{2}}{3}\right)+\frac{c(n)}{4 \pi^{2}}\left(\mu_{3}^{2}+\mu_{3_{5}}^{2}\right)\right] \boldsymbol{\omega},
\end{aligned}
$$

whereas in the non-Abelian currents it takes the form

$$
\begin{aligned}
\mathbf{J}_{3} & =\frac{c(n)}{2 \pi^{2}}\left(\mu \mu_{3_{5}}+\mu_{3} \mu_{5}\right) \boldsymbol{\omega}, \\
\mathbf{J}_{3_{5}} & =\frac{c(n)}{2 \pi^{2}}\left(\mu \mu_{3}+\mu_{3_{5}} \mu_{5}\right) \boldsymbol{\omega} .
\end{aligned}
$$

On the other hand, the energy current generated by the chiral magnetic and vortical effects reads

$$
\begin{aligned}
\mathbf{J}_{\epsilon}= & \frac{1}{2 \pi^{2}}\left[c(n) \mu_{3} \mu_{3_{5}}+n \mu \mu_{5}\right] \mathbf{B}+\frac{c(n)}{2 \pi^{2}}\left[\mu \mu_{3_{5}}+\mu_{3} \mu_{5}\right] \mathbf{B}_{3} \\
& +\frac{1}{4 \pi^{2}}\left[c(n)\left(\mu_{3}^{2}+\mu_{3_{5}}^{2}\right)+n\left(\mu^{2}+\mu_{5}^{2}\right)+\frac{n \pi^{2} T^{2}}{3}\right] \mathbf{B}_{5} \\
& +\frac{c(n)}{2 \pi^{2}}\left[\mu \mu_{3}+\mu_{5} \mu_{3_{5}}\right] \mathbf{B}_{3_{5}} \\
& +\frac{n}{6 \pi^{2}}\left[\mu_{5}\left(3 \mu^{2}+\mu_{5}^{2}\right)+\mu_{5} \pi^{2} T^{2}\right] \omega \\
& +\frac{c(n)}{2 \pi^{2}}\left[2 \mu \mu_{3} \mu_{3_{5}}+\mu_{5}\left(\mu_{3}^{2}+\mu_{3_{5}}^{2}\right)\right] \omega .
\end{aligned}
$$

\section{APPENDIX C: EQUATIONS OF MOTION}

In this Appendix we show the equations of motion for the holographic theory, introduced in Eq. (72). The MaxwellYang-Mills-Chern-Simons equations on the curved background are

$$
\nabla_{\mu} \mathcal{F}^{\mu \nu}-6 \lambda \epsilon^{v \rho \alpha \beta \gamma} \operatorname{Tr}\left(F_{\rho \alpha} F_{\beta \gamma}\right)=0,
$$

$$
\mathcal{D}_{\mu} G^{a, \mu \nu}-\frac{3}{2} \lambda \epsilon^{\nu \rho \alpha \beta \gamma} \operatorname{Tr}\left(s^{a} F_{\rho \alpha} F_{\beta \gamma}\right)=0 .
$$

After evaluating the ansatz (83), we find a set of equations for the Abelian and non-Abelian fields. First we show the equations of motion for the Abelian sector

$$
\begin{gathered}
\left(\frac{A_{t}^{\prime}}{r}+24 \lambda\left[c(n) \mathcal{Q}^{2} A_{z}^{3}+n B A_{z}\right]\right)^{\prime}=0, \\
\left(\frac{u(r) A_{z}^{\prime}}{r}+24 \lambda\left[c(n) \mathcal{Q}(r)^{2} A_{t}^{3}+n B A_{t}\right]\right)^{\prime}=0,
\end{gathered}
$$

where the prime stands for $\partial_{r}$. Notice that these equations can be integrated once, leading to

$$
\begin{aligned}
\frac{A_{t}^{\prime}}{r} & =4 \rho-24 \lambda\left[c(n) \mathcal{Q}^{2} A_{z}^{3}+n B A_{z}\right], \\
\frac{u(r) A_{z}^{\prime}}{r} & =-24 \lambda\left[c(n) \mathcal{Q}(r)^{2} A_{t}^{3}+n B A_{t}\right],
\end{aligned}
$$

where $\rho$ is an integration constant and the regularity at the horizon for $A_{z}$ forbids the presence of an extra integration constant. Now we show the equations for the non-Abelian sector, where the symmetry-breaking field $\mathcal{Q}(r)$ satisfies

$$
\left(\frac{u(r) \mathcal{Q}^{\prime}}{r}\right)^{\prime}+\left(\frac{\left(A_{t}^{3}\right)^{2}}{r u(r)}-\frac{\left(A_{z}^{3}\right)^{2}}{r}-\frac{\mathcal{Q}^{2}}{r}\right) \mathcal{Q}=0 .
$$

The rest of the equations read

$$
\left(\frac{u(r) A_{z}^{3^{\prime}}}{r}\right)^{\prime}-\frac{2 \mathcal{Q}^{2} A_{z}^{3}}{r}+24 \lambda c(n)\left[\mathcal{Q}^{2} A_{t}^{\prime}(r)+B A_{t}^{3^{\prime}}\right]=0
$$

$$
\left(\frac{A_{t}^{3^{\prime}}}{r}\right)^{\prime}-\frac{2 \mathcal{Q}^{2} A_{t}^{3}}{r u(r)}+24 \lambda c(n)\left[\mathcal{Q}^{2} A_{z}^{\prime}(r)+B A_{z}^{3^{\prime}}\right]=0 .
$$

[1] R. A. Bertlmann, Anomalies in Quantum Field Theory (Oxford University Press, Oxford, 2000).

[2] J. S. Bell and R. Jackiw, A PCAC puzzle: $\pi^{0} \rightarrow \gamma \gamma$ in the $\sigma$ model, Nuovo Cimento A 60, 47 (1969).

[3] S. L. Adler, Axial-vector vertex in spinor electrodynamics, Phys. Rev. 177, 2426 (1969).
[4] H. Nielsen and M. Ninomiya, The Adler-Bell-Jackiw anomaly and Weyl fermions in a crystal, Phys. Lett. B 130, 389 (1983).

[5] K. Fujikawa and H. Suzuki, Path Integrals and Quantum Anomalies (Oxford University Press, Oxford, 2004). 
[6] D. Kharzeev, J. Liao, S. Voloshin, and G. Wang, Chiral magnetic and vortical effects in high-energy nuclear collisions-A status report, Prog. Part. Nucl. Phys. 88, 1 (2016).

[7] W. A. Bardeen, Anomalous Ward identities in spinor field theories, Phys. Rev. 184, 1848 (1969).

[8] W. A. Bardeen and B. Zumino, Consistent and covariant anomalies in gauge and gravitational theories, Nucl. Phys. B 244, 421 (1984).

[9] R. Jackiw, When radiative corrections are finite but undetermined, Int. J. Mod. Phys. B 14, 2011 (2000).

[10] P. Goswami and S. Tewari, Axionic field theory of $(3+1)-$ dimensional Weyl semimetals, Phys. Rev. B 88, 245107 (2013).

[11] A. G. Grushin, Consequences of a condensed matter realization of Lorentz violating QED in Weyl semi-metals, Phys. Rev. D 86, 045001 (2012).

[12] A. Rebhan, A. Schmitt, and S. A. Stricker, Anomalies and the chiral magnetic effect in the Sakai-Sugimoto model, J. High Energy Phys. 01 (2010) 026.

[13] K. Landsteiner, E. Megías, and F. Peña-Benitez, Frequency dependence of the chiral vortical effect, Phys. Rev. D 90, 065026 (2014).

[14] A. A. Zyuzin and A. A. Burkov, Topological response in Weyl semimetals and the chiral anomaly, Phys. Rev. B 86, 115133 (2012).

[15] S. A. Parameswaran, T. Grover, D. A. Abanin, D. A. Pesin, and A. Vishwanath, Probing the Chiral Anomaly with Nonlocal Transport in Three-Dimensional Topological Semimetals, Phys. Rev. X 4, 031035 (2014).

[16] P. Goswami, J. H. Pixley, and S. Das Sarma, Axial anomaly and longitudinal magnetoresistance of a generic three-dimensional metal, Phys. Rev. B 92, 075205 (2015).

[17] X. Huang et al., Observation of the Chiral-Anomaly-Induced Negative Magnetoresistance in 3D Weyl Semimetal TaAs, Phys. Rev. X 5, 031023 (2015).

[18] Y.-Y. Wang, Q.-H. Yu, P.-J. Guo, K. Liu, and T.-L. Xia, Resistivity plateau and extremely large magnetoresistance in $\mathrm{NbAs}_{2}$ and $\mathrm{TaAs}_{2}$, Phys. Rev. B 94, 041103(R) (2016).

[19] G. Zheng et al., Transport evidence for the three-dimensional Dirac semimetal phase in $\mathrm{ZrTe}_{5}$, Phys. Rev. B 93, 115414 (2016).

[20] S. Wiedmann, A. Jost, B. Fauqué, J. van Dijk, M. J. Meijer, T. Khouri, S. Pezzini, S. Grauer, S. Schreyeck, C. Brüne, H. Buhmann, L. W. Molenkamp, and N. E. Hussey, Anisotropic and strong negative magnetoresistance in the three-dimensional topological insulator $\mathrm{Bi}_{2} \mathrm{Se}_{3}$, Phys. Rev. B 94, 081302(R) (2016).

[21] C.-L. Zhang et al., Signatures of the Adler-Bell-Jackiw chiral anomaly in a Weyl fermion semimetal, Nat. Commun. 7, 10735 (2016).

[22] F. Arnold et al., Negative magnetoresistance without welldefined chirality in the Weyl semimetal TaP, Nat. Commun. 7, 11615 (2016).

[23] N. Kikugawa et al., Interplanar coupling-dependent magnetoresistivity in high-purity layered metals, Nat. Commun. 7, 10903 (2016).

[24] Q. Li, D. E. Kharzeev, C. Zhang, Y. Huang, I. Pletikosić, A. V. Fedorov, R. D. Zhong, J. A. Schneeloch, G. D. Gu, and T. Valla, Chiral magnetic effect in $\mathrm{ZrTe}_{5}$, Nat. Phys. 12, 550 (2016).
[25] J. Gooth et al., Experimental signatures of the mixed axial-gravitational anomaly in the Weyl semimetal $\mathrm{NbP}$, Nature (London) 547, 324 (2017).

[26] C. Schindler et al., Observation of an anomalous heat current in GdPtBi, arXiv:1810.02300.

[27] C. Zhang et al., Room-temperature chiral charge pumping in Dirac semimetals, Nat. Commun. 8, 13741 (2017).

[28] A. Lucas, R. A. Davison, and S. Sachdev, Hydrodynamic theory of thermoelectric transport and negative magnetoresistance in Weyl semimetals, Proc. Natl. Acad. Sci. USA 113, 9463 (2016).

[29] J. L. Mañes, E. Megías, M. Valle, and M. Á. Vázquez-Mozo, Non-abelian anomalies and transport, EPJ Web Conf. 192, 00022 (2018)

[30] A. Avdoshkin, A. V. Sadofyev, and V. I. Zakharov, IR properties of chiral effects in pionic matter, Phys. Rev. D 97, 085020 (2018).

[31] G. Xu, H. Weng, Z. Wang, X. Dai, and Z. Fang, Chern Semimetal and the Quantized Anomalous Hall Effect in $\mathrm{HgCr}_{2} \mathrm{Se}_{4}$, Phys. Rev. Lett. 107, 186806 (2011).

[32] C. Fang, M. J. Gilbert, X. Dai, and B. A. Bernevig, Multi-Weyl Topological Semimetals Stabilized by Point Group Symmetry, Phys. Rev. Lett. 108, 266802 (2012).

[33] S.-M. Huang et al., New type of Weyl semimetal with quadratic double Weyl fermions, Proc. Natl. Acad. Sci. USA 113, 1180 (2016).

[34] Q. Liu and A. Zunger, Predicted Realization of Cubic Dirac Fermion in Quasi-One-Dimensional Transition-Metal Monochalcogenides, Phys. Rev. X 7, 021019 (2017).

[35] B.-J. Yang and N. Nagaosa, Classification of stable threedimensional Dirac semimetals with nontrivial topology, Nat. Commun. 5, 4898 (2014).

[36] Z.-M. Huang, J. Zhou, and S.-Q. Shen, Topological responses from chiral anomaly in multi-Weyl semimetals, Phys. Rev. B 96, 085201 (2017)

[37] L. Lepori, M. Burrello, and E. Guadagnini, Axial anomaly in multi-Weyl and triple-point semimetals, J. High Energy Phys. 06 (2018) 110.

[38] I. Amado, N. Lisker, and A. Yarom, Universal chiral conductivities for low temperature holographic superfluids, J. High Energy Phys. 06 (2014) 084.

[39] C. Copetti, J. Fernández-Pendás, and K. Landsteiner, Axial Hall effect and universality of holographic Weyl semi-metals, J. High Energy Phys. 02 (2017) 138.

[40] D. T. Son and P. Surowka, Hydrodynamics with Triangle Anomalies, Phys. Rev. Lett. 103, 191601 (2009).

[41] Y. Neiman and Y. Oz, Relativistic hydrodynamics with general anomalous charges, J. High Energy Phys. 03 (2011) 023 .

[42] A. Gynther, K. Landsteiner, F. Pena-Benitez, and A. Rebhan, Holographic anomalous conductivities and the chiral magnetic effect, J. High Energy Phys. 02 (2011) 110.

[43] K. Landsteiner, E. Megías, and F. Peña-Benitez, in Anomalous Transport from Kubo Formulae, edited by D. Kharzeev, K. Landsteiner, A. Schmitt, and H.-U. Yee, Lecture Notes in Physics Vol. 871 (Springer, Berlin, 2013), p. 433.

[44] B. Bradlyn, J. Cano, Z. Wang, M. G. Vergniory, C. Felser, R. J. Cava, and B. A. Bernevig, Beyond Dirac and Weyl fermions: Unconventional quasiparticles in conventional crystals, Science 353, aaf5037 (2016). 
[45] B. Bradlyn, L. Elcoro, J. Cano, M. G. Vergniory, Z. Wang, C. Felser, M. I. Aroyo, and B. A. Bernevig, Topological quantum chemistry, Nature (London) 547, 298 (2017).

[46] E. V. Gorbar, V. A. Miransky, I. A. Shovkovy, and P. O. Sukhachov, Origin of Bardeen-Zumino current in lattice models of Weyl semimetals, Phys. Rev. B 96, 085130 (2017).

[47] H.-F. Lü, Y.-H. Deng, S.-S. Ke, Y. Guo, and H.-W. Zhang, Quantum impurity in topological multi-Weyl semimetals, Phys. Rev. B 99, 115109 (2019).

[48] D. Sinha and K. Sengupta, Transport across junctions of a Weyl and a multi-Weyl semimetal, Phys. Rev. B 99, 075153 (2019).

[49] A. Menon and B. Basu, Hall transport of Landau quantized states in tilted multi-Weyl semimetals: Universality of anomalous vacuum contribution, arXiv:1901.06716.

[50] B. Roy and J. D. Sau, Magnetic catalysis and axionic charge density wave in Weyl semimetals, Phys. Rev. B 92, 125141 (2015).

[51] X. Li, B. Roy, and S. Das Sarma, Weyl fermions with arbitrary monopoles in magnetic fields: Landau levels, longitudinal magnetotransport, and density-wave ordering, Phys. Rev. B 94, 195144 (2016).

[52] N. P. Armitage, E. J. Mele, and A. Vishwanath, Weyl and Dirac semimetals in three-dimensional solids, Rev. Mod. Phys. 90, 015001 (2018).

[53] R. M. A. Dantas, F. Peña-Benitez, B. Roy, and P. Surówka, Magnetotransport in multi-Weyl semimetals: A kinetic theory approach, J. High Energy Phys. 12 (2018) 069.

[54] A. H. Castro Neto, F. Guinea, N. M. R. Peres, K. S. Novoselov, and A. K. Geim, The electronic properties of graphene, Rev. Mod. Phys. 81, 109 (2009).

[55] B. Roy, Classification of massive and gapless phases in bilayer graphene, Phys. Rev. B 88, 075415 (2013).

[56] M. Gell-Mann, Symmetries of baryons and mesons, Phys. Rev. 125, 1067 (1962).

[57] B. Roy, P. Goswami, and V. Juričić, Interacting Weyl fermions: Phases, phase transitions, and global phase diagram, Phys. Rev. B 95, 201102(R) (2017).

[58] S. Bera, J. D. Sau, and B. Roy, Dirty Weyl semimetals: Stability, phase transition, and quantum criticality, Phys. Rev. B 93, 201302(R) (2016).
[59] B. Roy, R.-J. Slager, and V. Juričić, Global Phase Diagram of a Dirty Weyl Liquid and Emergent Superuniversality, Phys. Rev. X 8, 031076 (2018).

[60] R.-J. Slager, V. Juričić, and B. Roy, Dissolution of topological Fermi arcs in a dirty Weyl semimetal, Phys. Rev. B 96, 201401(R) (2017).

[61] S. Nandy, S. Manna, D. Călugăru, and B. Roy, Generalized triple-component fermions: Lattice model, Fermi arcs, and anomalous transport, Phys. Rev. B 100, 235201 (2019).

[62] J. L. Manes, E. Megias, M. Valle, and M. A. Vazquez-Mozo, Non-Abelian anomalous (super)fluids in thermal equilibrium from differential geometry, J. High Energy Phys. 11 (2018) 076.

[63] D. E. Kharzeev and H. J. Warringa, Chiral magnetic conductivity, Phys. Rev. D 80, 034028 (2009).

[64] K. Landsteiner, E. Megías, and F. Pena-Benitez, Gravitational Anomaly and Transport, Phys. Rev. Lett. 107, 021601 (2011).

[65] A. Vilenkin, Macroscopic parity-violating effects: Neutrino fluxes from rotating black holes and in rotating thermal radiation, Phys. Rev. D 20, 1807 (1979).

[66] A. Vilenkin, Equilibrium parity-violating current in a magnetic field, Phys. Rev. D 22, 3080 (1980).

[67] N. Mueller and R. Venugopalan, Constructing phase space distributions with internal symmetries, Phys. Rev. D 99, 056003 (2019).

[68] R. Loganayagam and P. Surowka, Anomaly/transport in an ideal Weyl gas, J. High Energy Phys. 04 (2012) 097.

[69] K. Jensen, R. Loganayagam, and A. Yarom, Thermodynamics, gravitational anomalies and cones, J. High Energy Phys. 02 (2013) 88

[70] K. Landsteiner, Notes on anomaly induced transport, Acta Phys. Pol. B 47, 2617 (2016).

[71] M. M. Vazifeh and M. Franz, Electromagnetic Response of Weyl Semimetals, Phys. Rev. Lett. 111, 027201 (2013).

[72] J. Erdmenger, M. Haack, M. Kaminski, and A. Yarom, Fluid dynamics of R-charged black holes, J. High Energy Phys. 01 (2009) 055.

[73] N. Banerjee, J. Bhattacharya, S. Bhattacharyya, S. Dutta, R. Loganayagam, and P. Surowka, Hydrodynamics from charged black branes, J. High Energy Phys. 01 (2011) 094.

[74] U. Heinz, Kinetic Theory for Plasmas with Non-Abelian Interactions, Phys. Rev. Lett. 51, 351 (1983).

[75] D. F. Litim and C. Manuel, Semi-classical transport theory for non-Abelian plasmas, Phys. Rep. 364, 451 (2002). 